\title{
Anomalous Hall effect in Rashba two-dimensional electron systems based on narrow-band semiconductors: side-jump and skew scattering mechanisms
}

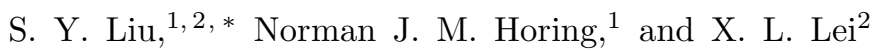 \\ ${ }^{1}$ Department of Physics and Engineering Physics, \\ Stevens Institute of Technology, Hoboken, New Jersey 07030, USA \\ ${ }^{2}$ Department of Physics, Shanghai Jiaotong University, 1954 Huashan Road, Shanghai 200030, China
}

\begin{abstract}
We employ a helicity-basis kinetic equation approach to investigate the anomalous Hall effect in two-dimensional narrow-band semiconductors considering both Rashba and extrinsic spin-orbit (SO) couplings, as well as a SO coupling directly induced by an external driving electric field. Taking account of long-range electron-impurity scattering up to the second Born approximation, we find that the various components of the anomalous Hall current fit into two classes: (a) side-jump and (b) skew scattering anomalous Hall currents. The side-jump anomalous Hall current involves contributions not only from the extrinsic SO coupling but also from the SO coupling due to the driving electric field. It also contains a component which arises from the Rashba SO coupling and relates to the off-diagonal elements of the helicity-basis distribution function. The skew scattering anomalous Hall effect arises from the anisotropy of the diagonal elements of the distribution function and it is a result of both the Rashba and extrinsic SO interactions. Further, we perform a numerical calculation to study the anomalous Hall effect in a typical InSb/AlInSb quantum well. The dependencies of the side-jump and skew scattering anomalous Hall conductivities on magnetization and on the Rashba SO coupling constant are examined.
\end{abstract}

PACS numbers: 73.50.Dn, 72.20.Fr, 73.63.Hs

\section{INTRODUCTION}

A nonvanishing magnetization in spin-split systems may lead to an extraordinary Hall current $\frac{1}{n}$ This so-called anomalous Hall effect (AHE) was first observed more than a century ago $\stackrel{2}{\underline{2}}$ but its complete understanding still remains a challenge today $\underline{3}^{3}$ Up to now, it has been made clear that the AHE arises essentially from spin-orbit (SO) interactions, which are the results of rapid movements of carriers in various electric fields, such as nuclear fields (e.g. the Dresselhaus SO coupling), electric fields associated with strains or gate biases (e.g. the Rashba SO coupling), or fields induced by electron-impurity scattering (extrinsic $\mathrm{SO}$ coupling), etc. $\stackrel{4}{=}$

In 1954, Karplus and Luttinger proposed, for the first time, a mechanism of the anomalous Hall effect $\underline{\underline{5}}$ This mechanism is associated with the spin-orbit interaction due to nuclear fields and yields to an anomalous Hall current (AHC) independent of any electron-impurity scattering. Recently, it has been reformulated by Jungwirth et al. within a framework of momentum-space Berry phase,,$\underline{6}$ and was used to explain the AHE in various ferromagnetic systems,

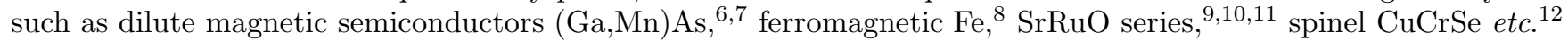

The AHE may also stem from a spin-orbit coupling induced by electron-impurity scattering, i.e. the extrinsic SO coupling $\underline{\underline{3}}$ It was found that there are two mechanisms responsible for this extrinsic AHE: a side-jump process proposed by Berger ${ }^{13}$ and a skew scattering given by Smit $\stackrel{14}{ }$ The side-jump AHE arises from a sidewise shift of the center of the electron wave packet and relates to an anomalous term in the current operator caused by the extrinsic SO coupling $\stackrel{13.15}{~ T h e ~ s k e w ~ s c a t t e r i n g ~ A H E ~ c o r r e s p o n d s ~ t o ~ a n ~ a n i s o t r o p i c ~ e n h a n c e m e n t ~ o f ~ t h e ~ w a v e ~ p a c k e t ~ d u e ~ t o ~ e l e c t r o n-~}$ impurity scattering and can be accounted for by considering the scattering in the second Born approximation $\underline{16}$ It was also clear that the side-jump anomalous Hall conductivity is independent of impurity density $n_{i}$, while the skewscattering one is proportional to $\left(n_{i}\right)^{-1}$. Recently, considering a short-range electron-impurity scattering, Crépieux, et al. presented a unified derivation of both the side-jump and skew scattering mechanisms within the framework of a formal Dirac equation for the electrons 17 The weak-localization corrections to these anomalous Hall currents have also been investigated $18,19,20,21$

The Rashba SO coupling in a two-dimensional (2D) electron system with magnetization can also give rise to a nonvanishing contribution to the Hall conductivity ${ }_{22,23.24}^{2}$ It was found that the anomalous Hall current due to the Rashba SO interaction consists of two terms: one of which is associated with all electron states below the Fermi surface and is independent of any electron-impurity scattering; and another one relating only to electrons near the Fermi surface, which is disorder-related but independent of impurity density 23.24 Liu and Lei have also clarified that, in the helicity basis, these two different contributions to anomalous Hall current in 2D Rashba semiconductors essentially arise from two distinct interband polarizations and they relate to two distinct parts of the off-diagonal elements of the distribution function ${ }^{24}$ In these studies, the Rashba SO coupling was considered nonperturbatively, but the extrinsic SO interaction was completely ignored. Such a treatment is valid only for 2D electron systems based on wide-band semiconductors. In 2D narrow-band semiconductors, such as InSb/AlInSb quantum wells (QWs), the 
coupling constant for extrinsic SO interaction is relatively large (for example, the extrinsic SO coupling constant, $\lambda$, is $\lambda=5.31 \mathrm{~nm}^{2}$ for InSb, while it is equal to $0.053 \mathrm{~nm}^{2}$ for $\left.\mathrm{GaAs}^{25}\right)$. Hence, to investigate the AHE in Rashba 2D systems based on narrow-band semiconductors, we have to consider not only the Rashba but also the extrinsic SO interactions.

In this paper, we employ a kinetic equation approach to investigate the AHE in Rashba 2D narrow-band semiconductors. We deal with the Rashba SO interaction in a nonperturbative way, while the extrinsic SO coupling is considered in the first order of the coupling constant $\lambda$. We also take account of the SO interaction induced directly by the external driving electric field, which, to our knowledge, was mentioned only by Nozières and Lewiner in the absence of Rashba SO coupling ${ }^{26}$ In our study, to investigate the skew-scattering AHE effect, we consider the electronimpurity scattering up to the second-Born approximation. It is found that various components of the anomalous Hall current can fit into two classes: the side-jump and skew scattering anomalous Hall currents. The side-jump AHC involves contributions from the extrinsic SO coupling and SO coupling induced by the driving electric field. It also contains a component which comes from the Rashba SO interaction and relates to the off-diagonal elements of the helicity-basis distribution function. The skew scattering AHC is associated with anisotropic diagonal components of the distribution function and stems from both the Rashba and extrinsic spin-orbit interactions. A numerical calculation of the anomalous Hall current in a InSb/AlInSb quantum well indicates that both the side-jump and skew scattering anomalous Hall currents are of the same order of magnitude, leading to complicated dependencies of the total anomalous Hall conductivity on magnetization and on the Rashba spin-orbit coupling constant. It is also clear that in the side-jump anomalous Hall current, the contribution from SO coupling due to the driving electric field is dominant for small magnetization.

This paper is organized as follows. In Sec. II, we derive the kinetic equation for the nonequilibrium distribution function considering long-range electron-impurity scattering up to the second Born approximation. The solution of this equation to first order of the extrinsic spin-orbit coupling is presented. We also discuss the various components of the side-jump and skew scattering anomalous Hall currents. In Sec. III, we perform a numerical calculation to investigate the anomalous Hall effect in a InSb/AlInSb quantum well. Finally, we review our results in Sec. IV. The detailed form of the scattering term of the kinetic equation is presented in an Appendix.

\section{FORMALISM}

\section{A. Hamiltonian and current operator}

We consider a Rashba quasi-two-dimensional narrow-band semiconductor in the $x-y$ plane. When a homogeneous magnetization $\mathbf{M} \equiv(0,0, M)$ induced by a weak magnetic field $B, M=g \mu_{B} B$ ( $g$ is the effective g-factor and $\mu_{B}$ is the Bohr magneton), and a uniform in-plane dc electric field $\mathbf{E}$ are present, the Hamiltonian of an electron with momentum $\mathbf{p} \equiv\left(p_{x}, p_{y}\right) \equiv\left(p \cos \phi_{\mathbf{p}}, p \sin \phi_{\mathbf{p}}\right)$ can be written as

$$
\check{H}=\check{H}_{0}+\check{H}_{\mathrm{imp}}+\check{H}_{E} .
$$

$\check{H}_{0}$ is the free electron Hamiltonian given by

$$
\check{H}_{0}=\varepsilon_{p}+\alpha\left(\sigma^{x} p_{y}-\sigma^{y} p_{x}\right)-M \sigma^{z}
$$

where $\alpha$ is the Rashba SO coupling constant, $\sigma^{l}(l=x, y, z)$ are the Pauli matrices, and $\varepsilon_{p}=p^{2} / 2 m^{*}$ with $m^{*}$ as the electron effective mass. By a local unitary spinor transformation, $U_{\mathbf{p}}$,

$$
U_{\mathbf{p}}=\frac{1}{\sqrt{2 \lambda_{p}}}\left(\begin{array}{c}
\sqrt{\lambda_{p}+M} \\
i \mathrm{e}^{i \phi_{\mathbf{p}}} \sqrt{\lambda_{p}-M}-i \mathrm{e}^{i \phi_{\mathbf{p}}} \sqrt{\lambda_{p}+M}
\end{array}\right)
$$

Hamiltonian (2) can be diagonalized as $\hat{H}_{0} \equiv U_{\mathbf{p}}^{+} \check{H}_{0} U_{\mathbf{p}}=\operatorname{diag}\left(\varepsilon_{1}(p), \varepsilon_{2}(p)\right)$ with $\varepsilon_{\mu}(p)=p^{2} / 2 m^{*}+(-1)^{\mu} \lambda_{p}(\mu=1,2$ and $\left.\lambda_{p} \equiv \sqrt{M^{2}+\alpha^{2} p^{2}}\right)$ as dispersion relations of two spin-orbit-coupled bands.

Since the extrinsic spin-orbit coupling in narrow-band semiconductors cannot be ignored, the Hamiltonian $\check{H}_{\text {imp }}$, which describes the electron-impurity interaction, should contain not only an ordinary scattering potential term but also a term related to the extrinsic SO coupling:

$$
\check{H}_{\mathrm{imp}}=\sum_{i}\left\{V\left(\left|\mathbf{r}-\mathbf{R}_{i}\right|\right)-\lambda\left[\sigma \times \nabla V\left(\left|\mathbf{r}-\mathbf{R}_{i}\right|\right)\right] \cdot \mathbf{p}\right\},
$$

where $\mathbf{r}$ and $\mathbf{R}_{i}$, respectively, are the coordinates of the electron and impurity, $V(r)$ is the electron-impurity scattering potential, and $\lambda$ is a spin-orbit coupling constant depending on the intrinsic semiconductor parameters, such as energy 
gap $E_{0}$, spin-orbit splitting $\Delta_{\mathrm{SO}}$, and matrix element of the momentum operator between the conduction and valence bands $P: \lambda=\left[1 / E_{0}^{2}-1 /\left(E_{0}+\Delta_{\mathrm{SO}}\right)^{2}\right] P^{2} / 3{ }^{27} H_{E}$ describes the application of the external electric field, and, in the Coulomb gauge, it can be written as

$$
\check{H}_{E}=-e \mathbf{E} \cdot \mathbf{r}-\lambda[\sigma \times \mathbf{E}] \cdot \mathbf{p} .
$$

In Eq. (5), we have considered the effect of the spin-orbit coupling directly induced by the external driving dc electric field.

From Hamiltonian (1), it follows that, in spin basis, the single-particle current operator, $\check{\mathbf{j}}(\mathbf{p})$, can be written as

$$
\check{j}_{l}(\mathbf{p})=\check{j}_{l}^{\mathrm{f}}(\mathbf{p})+\check{j}_{l}^{\mathrm{imp}}(\mathbf{p})+\check{j}_{l}^{E}(\mathbf{p})
$$

with $l=x, y$. The term $\check{j}_{l}^{\mathrm{f}}(\mathbf{p})$ comes from the free-electron Hamiltonian $\check{H}_{0}: \check{j}_{l}^{\mathrm{f}}(\mathbf{p})=e p_{l} / m^{*}-\alpha \epsilon_{l m z} \sigma^{m}(m=x, y$ and $\epsilon_{l m z}$ is the totally antisymmetric tensor), while $\breve{j}_{l}^{\text {imp }}(\mathbf{p})$ comes from the SO coupling term of $\check{H}_{\text {imp }}$ and takes the form $(n=x, y)$

$$
\check{j}_{l}^{\mathrm{imp}}(\mathbf{p})=i \lambda e \sum_{\mathbf{k}, i} V_{\mathbf{p}-\mathbf{k}} \mathrm{e}^{i \mathbf{R}_{i} \cdot(\mathbf{k}-\mathbf{p})}\left[\epsilon_{l m n}\left(k_{m}-p_{m}\right) \sigma^{n}\right]
$$

The term $\breve{j}_{l}^{E}(\mathbf{p})$ arises from the spin-orbit coupling directly induced by the external driving electric field and it is given by

$$
\check{j}_{l}^{E}(\mathbf{p})=-\lambda e^{2} \epsilon_{l m n} \sigma^{m} E_{n} .
$$

Taking the statistical ensemble average, we find that the observed net current, J, consists of three components:

$$
J_{l}=J_{l}^{\mathrm{f}}+J_{l}^{\mathrm{imp}}+J_{l}^{E}
$$

$J_{l}^{\mathrm{f}, E}$ is determined by $J_{l}^{\mathrm{f}, E}=\sum_{\mathbf{p}} \operatorname{Tr}\left[\check{j}_{l}^{\mathrm{f}, E}(\mathbf{p}) \check{\rho}(\mathbf{p})\right]$, with $\check{\rho}(\mathbf{p})$ as the distribution function in the spin basis: $\check{\rho}_{\mu \nu}(\mathbf{p})=<$ $\check{\psi}_{\nu \mathbf{p}}^{+} \check{\psi}_{\mu \mathbf{p}}>\left(\check{\psi}_{\mu \mathbf{p}}^{+}\right.$and $\check{\psi}_{\mu \mathbf{p}}$, respectively, are the spin-basis electron creation and annihilation operators $)$. $J_{l}^{\text {imp }}$ arises from the current operator term $j_{l}^{\text {imp }}(\mathbf{p})$ and takes the form

$$
J_{l}^{\mathrm{imp}}=i \lambda e \sum_{\mathbf{p}, \mathbf{k}, i, \mu, \nu} V_{\mathbf{p}-\mathbf{k}} \mathrm{e}^{i \mathbf{R}_{i} \cdot(\mathbf{k}-\mathbf{p})}\left\{<\check{\psi}_{\nu \mathbf{p}}^{+} \check{\psi}_{\mu \mathbf{k}}>\left[\epsilon_{l m n}\left(k_{m}-p_{m}\right) \sigma_{\nu \mu}^{n}\right]\right\}
$$

Obviously, to determine $J_{l}^{\text {imp }}$, one has to analyze the function $<\check{\psi}_{\nu \mathbf{p}}^{+} \check{\psi}_{\mu \mathbf{k}}>$.

Without loss of generality, we study here the anomalous Hall current flow along the $x$-axis when the electric field is applied along the $y$-direction, i.e. $\mathbf{E}=(0, E, 0)$. In helicity basis, the current $J_{x}^{E}$ can be written as

$$
J_{x}^{E}=\lambda e^{2} E \sum_{\mathbf{p}}\left[\hat{\rho}_{11}(\mathbf{p})-\hat{\rho}_{22}(\mathbf{p})\right]
$$

with $\hat{\rho}_{\mu \nu}(\mathbf{p})(\mu, \nu=1,2)$ defined as the elements of the helicity-basis distribution function related to the spin-basis distribution function by $\hat{\rho}(\mathbf{p})=U_{\mathbf{p}}^{+} \check{\rho}(\mathbf{p}) U_{\mathbf{p}} . J_{x}^{\mathrm{f}}$ can be expressed as a sum of the contributions from the diagonal and off-diagonal elements of the helicity-basis distribution function, $J_{x}^{\mathrm{fd}}$ and $J_{x}^{\mathrm{fo}}$ :

$$
J_{x}^{\mathrm{f}}=J_{x}^{\mathrm{fd}}+J_{x}^{\mathrm{fo}}
$$

with

$$
J_{x}^{\mathrm{fd}}=e \sum_{\mathbf{p}}\left\{\left(\frac{1}{m^{*}}-\frac{\alpha^{2}}{\lambda_{p}}\right) p \cos \phi_{\mathbf{p}} \hat{\rho}_{11}(\mathbf{p})+\left(\frac{1}{m^{*}}+\frac{\alpha^{2}}{\lambda_{p}}\right) p \cos \phi_{\mathbf{p}} \hat{\rho}_{22}(\mathbf{p})\right\}
$$

and

$$
J_{x}^{\mathrm{fo}}=e \sum_{\mathbf{p}}\left\{\frac{2 \alpha M}{\lambda_{p}} \cos \phi_{\mathbf{p}} \operatorname{Re} \hat{\rho}_{12}(\mathbf{p})+2 \alpha \sin \phi_{\mathbf{p}} \operatorname{Im} \hat{\rho}_{12}(\mathbf{p})\right\}
$$


From Eq. (14), we can see that the nonvanishing contribution to $J_{x}^{\text {fo }}$ comes from the component of the real part of $\hat{\rho}_{12}(\mathbf{p})$, depending on the momentum angle through $\cos \phi_{\mathbf{p}}: \operatorname{Re}\left[(\hat{\rho})_{12}(\mathbf{p})\right]=\xi(p) \cos \phi_{\mathbf{p}}+\ldots$, and from the component of the imaginary part of $\hat{\rho}_{12}(\mathbf{p})$, involving $\sin \phi_{\mathbf{p}}: \operatorname{Im}\left[(\hat{\rho})_{12}(\mathbf{p})\right]=\zeta(p) \sin \phi_{\mathbf{p}}+\ldots$. As a result, $J_{x}^{\text {fo }}$ can be rewritten as

$$
J_{x}^{\mathrm{fo}}=2 \alpha e^{2} E \sum_{\mathbf{p}}\left[\frac{M \xi(p)}{\lambda_{p}} \cos ^{2} \phi_{\mathbf{p}}+\zeta(p) \sin ^{2} \phi_{\mathbf{p}}\right] .
$$

Note that these $J_{x}$ components, $J_{x}^{\mathrm{f}}, J_{x}^{\mathrm{imp}}$, and $J_{x}^{E}$, can fit into two classes: (a) side-jump and (b) skew-scattering anomalous Hall currents. We know that the side-jump AHE originates from the shift of the electron wave-packet center towards the direction transverse to the driving electric field. Such a shift is reflected by those current operator components involving the antisymmetric tensor $\epsilon_{l m n}: \breve{j}_{l}^{\text {imp }}, \breve{j}_{l}^{E}$ and the term in $\breve{j}_{l}^{\mathrm{f}}$ associated with the Rashba spinorbit coupling. Correspondingly, the observed side-jump anomalous Hall current $J_{x}^{\text {sj }}$ is a sum of $J_{x}^{\text {imp }}, J_{x}^{E}$ and $J_{x}^{\text {fo }}$ : $J_{x}^{\mathrm{sj}}=J_{x}^{\mathrm{fo}}+J_{x}^{\mathrm{imp}}+J_{x}^{E}$. From Eq. (13), we see that the remaining $J_{x}$ component, $J_{x}^{\mathrm{fd}}$, becomes nonvanishing if there exists a component of $\hat{\rho}_{\mu \mu}(\mathbf{p})$ depending on the angle of momentum through $\cos \phi_{\mathbf{p}}$. This implies that $J_{x}^{\mathrm{fd}}$ results from an anisotropy due to electron-impurity scattering and hence it just is a component of $J_{x}^{\mathrm{ss}}: J_{x}^{\mathrm{ss}}=J_{x}^{\mathrm{fd}}$.

\section{B. Kinetic equation and its solution}

Obviously, in order to carry out the calculation of the anomalous Hall current, it is necessary to determine the electron distribution function. Under homogeneous and steady-state conditions (averaging over a uniform impurity distribution), the helicity-basis distribution function, $\hat{\rho}(\mathbf{p})$, obeys a kinetic equation written in the form

$$
e \mathbf{E} \cdot \nabla_{\mathbf{p}} \hat{\rho}(\mathbf{p})-e \mathbf{E} \cdot\left[\hat{\rho}(\mathbf{p}), U_{\mathbf{p}}^{+} \nabla_{\mathbf{p}} U_{\mathbf{p}}\right]-i \lambda e \mathbf{E} \cdot(\mathbf{p} \times \mathbf{n})\left[U_{\mathbf{p}}^{+} \sigma^{z} U_{\mathbf{p}}, \hat{\rho}(\mathbf{p})\right]+i\left[H_{0}, \hat{\rho}(\mathbf{p})\right]=-\hat{I},
$$

where $\mathbf{n}$ is a unit vector along $z$-axis and $\hat{I}$ is a scattering term determined by

$$
\hat{I}=\int \frac{d \omega}{2 \pi}\left[\hat{\Sigma}^{r}(\mathbf{p}, \omega) \hat{\mathrm{G}}^{<}(\mathbf{p}, \omega)+\hat{\Sigma}^{<}(\mathbf{p}, \omega) \hat{\mathrm{G}}^{a}(\mathbf{p}, \omega)-\hat{\mathrm{G}}^{r}(\mathbf{p}, \omega) \hat{\Sigma}^{<}(\mathbf{p}, \omega)-\hat{\mathrm{G}}^{<}(\mathbf{p}, \omega) \hat{\Sigma}^{a}(\mathbf{p}, \omega)\right] .
$$

$\hat{\mathrm{G}}^{r, a,<}(\mathbf{p}, \omega)$ and $\hat{\Sigma}^{r, a,<}(\mathbf{p}, \omega)$ are, respectively, the helicity-basis nonequilibrium Green's functions and self-energies. Eq. (16) is derived from the Dyson equation of the spin-basis nonequilibrium lesser Green's function by applying the unitary transformation $U_{\mathbf{p}}$.

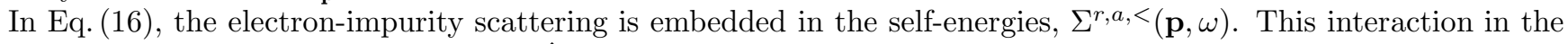
helicity basis is described by a potential, $\hat{\mathcal{V}}_{\mathbf{p k}}$, which can be written as

$$
\hat{\mathcal{V}}_{\mathbf{p k}}=U_{\mathbf{p}}^{+} V(\mathbf{p}-\mathbf{k}) U_{\mathbf{k}}+i \lambda U_{\mathbf{p}}^{+}[\mathbf{n} \cdot(\mathbf{p} \times \mathbf{k})] V(\mathbf{p}-\mathbf{k}) U_{\mathbf{k}}
$$

In terms of the Feynman diagrams, $\hat{\mathcal{V}}_{\mathbf{p k}}$ is denoted by two different interaction vertices: ordinary and anomalous vertices, which, respectively, are depicted in Figs. 1(a) and 1(b). Since electron-impurity scattering will be considered up to the second Born approximation, it is convenient to express the self-energies by means of generalized T-matrices, $\hat{T}_{\mathbf{p k}}^{r, a}(\omega)$, which obey the equations 28

$$
\hat{T}_{\mathbf{p k}}^{r, a}(\omega)=\hat{\mathcal{V}}_{\mathbf{p k}}+\sum_{\mathbf{q}} \hat{\mathcal{V}}_{\mathbf{p q}} \hat{\mathrm{G}}^{r, a}(\mathbf{q}, \omega) \hat{T}_{\mathbf{q k}}^{r, a}(\omega)
$$

[These equations are exhibited in terms of Feynman diagrams in Fig. 1(c).] Thus, the lesser self-energy can be written $\operatorname{as}^{28}$

$$
\hat{\Sigma}^{<}(\mathbf{p}, \omega)=n_{i} \sum_{\mathbf{k}} \hat{T}_{\mathbf{p k}}^{r}(\omega) \hat{\mathrm{G}}^{<}(\mathbf{k}, \omega) \hat{T}_{\mathbf{k p}}^{a}(\omega)
$$

while the retarded and advanced self-energies take the forms

$$
\hat{\Sigma}^{r, a}(\mathbf{p}, \omega)=n_{i} \hat{T}_{\mathbf{p p}}^{r, a}(\omega)
$$

In present paper, we restrict our considerations to the linear response regime. In connection with this, all the functions, such as the nonequilibrium Green's functions, self-energies, and distribution function, can be expressed as sums of two terms: $A=A_{0}+A_{1}$, with $A$ representing the Green's functions, self-energies or distribution function. $A_{0}$ 
and $A_{1}$, respectively, are the unperturbed part and the linear electric field part of $A$. In this way, the kinetic equation for the linear electric field part of the distribution, $\hat{\rho}_{1}(\mathbf{p})$, can be written as

$$
e \mathbf{E} \cdot \nabla_{\mathbf{p}} \hat{\rho}_{0}(\mathbf{p})-e \mathbf{E} \cdot\left[\hat{\rho}_{0}(\mathbf{p}), U_{\mathbf{p}}^{+} \nabla_{\mathbf{p}} U_{\mathbf{p}}\right]-i \lambda e \mathbf{E} \cdot(\mathbf{p} \times \mathbf{n})\left[U_{\mathbf{p}}^{+} \sigma^{z} U_{\mathbf{p}}, \hat{\rho}_{0}(\mathbf{p})\right]+i\left[\hat{H}_{0}, \hat{\rho}_{1}(\mathbf{p})\right]=-\hat{I}^{(1)},
$$

with $\hat{I}^{(1)}$ as the linear electric field part of the collision term $\hat{I}$ :

$$
\hat{I}^{(1)}=\int \frac{d \omega}{2 \pi}\left[\hat{\Sigma}_{1}^{<}(\mathbf{p}, \omega) \hat{\mathrm{G}}_{0}^{a}(\mathbf{p}, \omega)-\hat{\mathrm{G}}_{1}^{<}(\mathbf{p}, \omega) \hat{\Sigma}_{0}^{a}(\mathbf{p}, \omega)+\hat{\Sigma}_{0}^{r}(\mathbf{p}, \omega) \hat{\mathrm{G}}_{1}^{<}(\mathbf{p}, \omega)-\hat{\mathrm{G}}_{0}^{r}(\mathbf{p}, \omega) \hat{\Sigma}_{1}^{<}(\mathbf{p}, \omega)\right] .
$$

We note that, here, the effect of $\hat{\mathrm{G}}_{1}^{r, a}(\mathbf{p}, \omega)$ on distribution function has been ignored because these linear electric parts of the retarded and advanced Green's functions lead to a collisional broadening effect on $\hat{\rho}_{1}(\mathbf{p})$, which plays a secondary role in transport studies.

To further simplify Eq. (22), we employ a two-band generalized Kadanoff-Baym ansatz (GKBA).29.30 This ansatz, which expresses the lesser Green's function through the Wigner distribution function, has been proven sufficiently accurate to analyze transport and optical properties in semiconductors ${ }^{31}$ To first order in the dc field strength, the GKBA reads,

$$
\hat{\mathrm{G}}_{1}^{<}(\mathbf{p}, \omega)=-\hat{\mathrm{G}}_{0}^{r}(\mathbf{p}, \omega) \hat{\rho}_{1}(\mathbf{p})+\hat{\rho}_{1}(\mathbf{p}) \hat{\mathrm{G}}_{0}^{a}(\mathbf{p}, \omega),
$$

where the retarded and advanced Green's functions in helicity basis are diagonal matrices: $\hat{\mathrm{G}}_{0}^{r, a}(\mathbf{p}, \omega)=\operatorname{diag}[(\omega-$ $\left.\left.\varepsilon_{1}(p) \pm i \delta\right)^{-1},\left(\omega-\varepsilon_{2}(p) \pm i \delta\right)^{-1}\right]$. Note that the helicity-basis equilibrium distribution is also diagonal, $\hat{\rho}_{0}(\mathbf{p})=$ $\operatorname{diag}\left[n_{\mathrm{F}}\left(\varepsilon_{1}(p)\right), n_{\mathrm{F}}\left(\varepsilon_{2}(p)\right)\right]$ with $n_{\mathrm{F}}(\omega)$ as the Fermi function.

Since our studies are concerned with electron transport within the diffusive regime, it is sufficient to study the lowest order of the distribution function in the impurity-density expansion. From the diagonal parts of Eq. (22), we see that the leading order of the diagonal $\hat{\rho}_{1}(\mathbf{p})$ elements, $\left(\hat{\rho}_{1}\right)_{\mu \mu}(\mathbf{p})$, is proportional to $\left(n_{i}\right)^{-1}$. These diagonal elements of the distribution function give rise to off-diagonal elements of the scattering term $\hat{I}^{(1)}$, which are independent of the impurity density. From the fact that the left-hand side of the off-diagonal parts of Eq. (22) involves the term $i\left[\hat{H}_{0}, \hat{\rho}_{1}(\mathbf{p})\right]$ proportional to the off-diagonal elements of the distribution function:

$$
i\left[\hat{H}_{0}, \hat{\rho}_{1}(\mathbf{p})\right]=-2 i \lambda_{p}\left(\begin{array}{cc}
0 & \left(\hat{\rho}_{1}\right)_{12}(\mathbf{p}) \\
\left(\hat{\rho}_{1}\right)_{21}(\mathbf{p}) & 0
\end{array}\right)
$$

it follows that the leading order of the off-diagonal elements of $\hat{\rho}_{1}(\mathbf{p})$ should be of order $\left(n_{i}\right)^{0}$, i.e. independent of the impurity density. Note that the contributions to $\hat{I}^{(1)}$ from such off-diagonal elements of $\hat{\rho}_{1}(\mathbf{p})$ are linear in the impurity density and hence can be ignored, while the contributions to $\hat{I}^{(1)}$ from the diagonal elements, $\left(\hat{\rho}_{1}\right)_{\mu \mu}(\mathbf{p})$, are independent of $n_{i}$ and become dominant. Thus, to the lowest-order of the $n_{i}$-power expansion, $\hat{I}^{(1)}$ effectively involves only the diagonal elements of the distribution function.

From Eq. (22) we see that the driving force of the kinetic equation can be classified into two classes: diagonal $e \mathbf{E} \cdot \nabla_{\mathbf{p}} \hat{\rho}_{0}$, and off-diagonal $-e \mathbf{E} \cdot\left[\hat{\rho}_{0}(\mathbf{p}), U_{\mathbf{p}}^{+} \nabla_{\mathbf{p}} U_{\mathbf{p}}\right]$ and $-i \lambda e \mathbf{E} \cdot(\mathbf{p} \times \mathbf{n})\left[U_{\mathbf{p}}^{+} \sigma^{z} U_{\mathbf{p}}, \hat{\rho}_{0}(\mathbf{p})\right]$. In connection with this, we may formally split the kinetic equation into two equations with $\hat{\rho}_{1}(\mathbf{p})=\hat{\rho}_{1}^{I}(\mathbf{p})+\hat{\rho}_{1}^{I I}(\mathbf{p})$ as

$$
\begin{gathered}
e \mathbf{E} \cdot \nabla_{\mathbf{p}} \hat{\rho}_{0}(\mathbf{p})+i\left[\hat{H}_{0}, \hat{\rho}_{1}^{I}(\mathbf{p})\right]=-\hat{I}^{(1)} \\
-e \mathbf{E} \cdot\left[\hat{\rho}_{0}(\mathbf{p}), U_{\mathbf{p}}^{+} \nabla_{\mathbf{p}} U_{\mathbf{p}}\right]-i \lambda e \mathbf{E} \cdot(\mathbf{p} \times \mathbf{n})\left[U_{\mathbf{p}}^{+} \sigma^{z} U_{\mathbf{p}}, \hat{\rho}_{0}(\mathbf{p})\right]+i\left[\hat{H}_{0}, \hat{\rho}_{1}^{I I}(\mathbf{p})\right]=0 .
\end{gathered}
$$

From Eq. (27) it is evident that $\hat{\rho}_{1}^{I I}(\mathbf{p})$ has null diagonal elements. Since $\hat{I}^{(1)}$ depends only on the diagonal elements of the distribution function, $\hat{\rho}_{1}^{I}(\mathbf{p})$ and $\hat{\rho}_{1}^{I I}(\mathbf{p})$ can be approximately determined independently of one another by Eqs. (26) and (27).

Substituting the explicit forms of $\hat{\mathrm{G}}_{0}^{r, a}(\mathbf{p}, \omega)$ into Eq. (23) and considering Eq. (24), the elements of the linear electric-field scattering term, $\hat{I}^{(1)}$, can be written as

$$
\begin{aligned}
\hat{I}_{\mu \mu}^{(1)}= & -2 \operatorname{Im}\left[\left(\hat{T}_{\mathbf{p p}}^{r}\right)_{\mu \mu}\left(\varepsilon_{\mu}(p)\right)\right]\left(\hat{\rho}_{1}^{I}\right)_{\mu \mu}(\mathbf{p}) \\
& -2 \pi \sum_{\mathbf{k}} \delta\left[\varepsilon_{\mu}(p)-\varepsilon_{\mu}(k)\right]\left(\hat{T}_{\mathbf{p k}}^{r}\right)_{\mu \mu}\left(\varepsilon_{\mu}(k)\right)\left(\hat{T}_{\mathbf{k p}}^{a}\right)_{\mu \mu}\left(\varepsilon_{\mu}(k)\right)\left(\hat{\rho}_{1}^{I}\right)_{\mu \mu}(\mathbf{k}) \\
& -2 \pi \sum_{\mathbf{k}} \delta\left[\varepsilon_{\mu}(p)-\varepsilon_{\bar{\mu}}(k)\right]\left(\hat{T}_{\mathbf{p k}}^{r}\right)_{\mu \bar{\mu}}\left(\varepsilon_{\bar{\mu}}(k)\right)\left(\hat{T}_{\mathbf{k p}}^{a}\right)_{\bar{\mu} \mu}\left(\varepsilon_{\bar{\mu}}(k)\right)\left(\hat{\rho}_{1}^{I}\right)_{\bar{\mu} \bar{\mu}}(\mathbf{k}),
\end{aligned}
$$


(a)

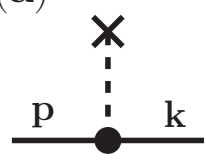

(b)

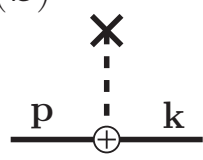

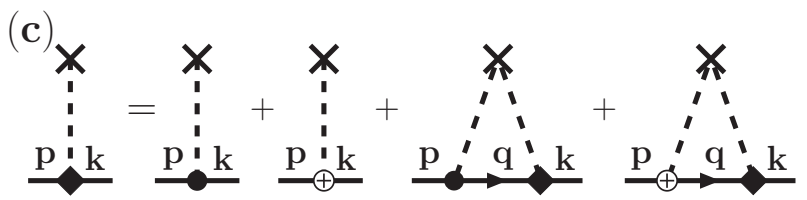
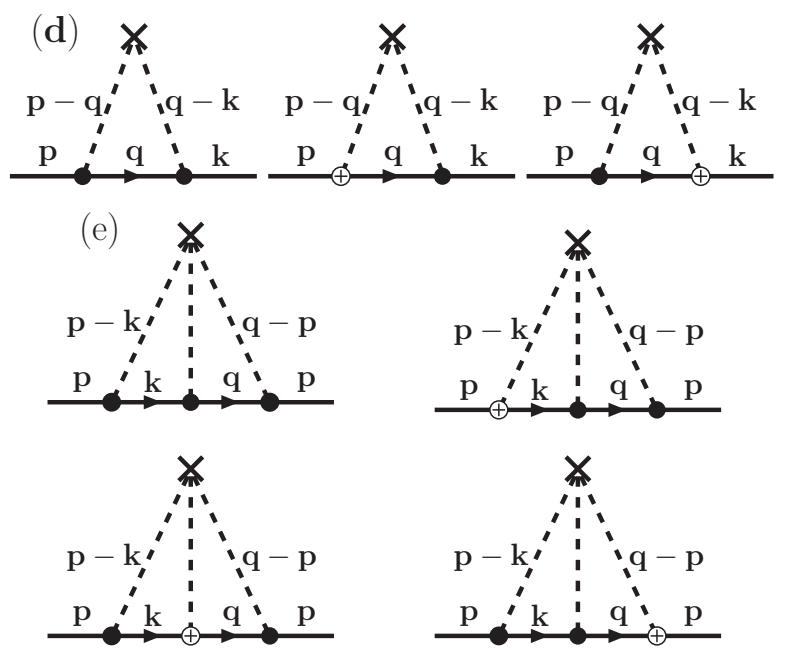

FIG. 1: Feynman diagrams for electron-impurity scattering. (a) and (b) show the interaction vertices, which, respectively, correspond to the original scattering potential and the potential due to the extrinsic SO coupling. (c) is the equation for $T$-matrix. (d) and (e), respectively, are Feynman diagrams for the $T$-matrix in the first and second Born approximations up to the first order of the spin-orbit coupling constant, $\lambda$.

and

$$
\begin{aligned}
\hat{I}_{\mu \bar{\mu}}^{(1)}= & i\left(\hat{T}_{\mathbf{p p}}^{r}\right)_{\mu \bar{\mu}}\left(\varepsilon_{\bar{\mu}}(p)\right)\left(\hat{\rho}_{1}^{I}\right)_{\bar{\mu} \bar{\mu}}(\mathbf{p})-i\left(\hat{T}_{\mathbf{p p}}^{a}\right)_{\mu \bar{\mu}}\left(\varepsilon_{\mu}(p)\right)\left(\hat{\rho}_{1}^{I}\right)_{\mu \mu}(\mathbf{p}) \\
& +\sum_{\mathbf{k}, \nu}\left\{\left(\hat{T}_{\mathbf{p k}}^{r}\right)_{\mu \nu}\left(\varepsilon_{\nu}(k)\right)\left(\hat{T}_{\mathbf{k p}}^{a}\right)_{\nu \bar{\mu}}\left(\varepsilon_{\nu}(k)\right)\left(\hat{\rho}_{1}^{I}\right)_{\nu \nu}(\mathbf{k})\left[\left(\hat{\mathrm{G}}_{0}^{a}\right)_{\bar{\mu} \bar{\mu}}\left(\mathbf{p}, \varepsilon_{\nu}(k)\right)-\left(\hat{\mathrm{G}}_{0}^{r}\right)_{\mu \mu}\left(\mathbf{p}, \varepsilon_{\nu}(k)\right)\right]\right\},
\end{aligned}
$$

with $\mu=1,2$ and $\bar{\mu}=3-\mu$.

Further, as in all previous studies, we consider the anomalous Hall current only to the first order of the spinorbit coupling constant $\lambda$. Thus, the scattering term $\hat{I}^{(1)}$ and hence the $T$-matrix may be considered only in the lowest- and first-order of $\lambda$. On the other hand, we will evaluate the diagonal elements of $\hat{I}^{(1)}$ up to the second-Born approximation, but its off-diagonal elements only in the first-Born approximation. It is widely accepted that, in usual cases, the self-consistent first Born approximation is sufficiently accurate to analyze transport in diffusive regime (correspondingly, the scattering term may be considered only in the first-Born approximation). In our studies, $\hat{I}_{\mu \mu}^{(1)}$ is evaluated up to the second Born approximation because we should account for the skew scattering AHC associated only with the diagonal elements of the distribution function. Under these considerations, we need to analyze only the Feynman diagrams depicted in Figs. 1(a), 1(b), 1(d), and 1(e). Substituting the T-matrix obtained from these diagrams, we obtain the explicit form of the scattering term $\hat{I}^{(1)}$, which is presented in the Appendix.

Considering the elastic features of the electron-impurity scattering, Eq. (26) can be solved analytically. We know that $\hat{I}^{(1)}$ does not involve the off-diagonal elements of $\hat{\rho}_{1}^{I}(\mathbf{p})$. Hence, the diagonal $\hat{\rho}_{1}^{I}(\mathbf{p})$ elements can be determined from the diagonal parts of Eq. (26), while its off-diagonal elements are given by substituting the obtained results for $\left(\hat{\rho}_{1}^{I}\right)_{\mu \mu}(\mathbf{p})$ into the off-diagonal parts of Eq. (26). We assume that the solution $\hat{\rho}_{1}^{I}(\mathbf{p})$ can be expressed as $\hat{\rho}_{1}^{I}(\mathbf{p})=\hat{\mathcal{R}}_{0}(\mathbf{p})+\hat{\mathcal{R}}_{1}(\mathbf{p})$ with $\hat{\mathcal{R}}_{0}(\mathbf{p})$ and $\hat{\mathcal{R}}_{1}(\mathbf{p})$ as the lowest- and first-order terms of $\hat{\rho}_{1}^{I}(\mathbf{p})$ in the $\lambda$ expansion. In the lowest-order of $\lambda$, the 
diagonal elements of the distribution function, $\hat{\mathcal{R}}_{0 \mu \mu}(\mathbf{p})$, are determined by

$$
e \mathbf{E} \cdot \nabla_{\mathbf{p}} \hat{\rho}_{0 \mu \mu}(\mathbf{p})=-\mathcal{I}_{\mu}^{a}\left[\hat{\mathcal{R}}_{0}\right]-\mathcal{I}_{\mu}^{c}\left[\hat{\mathcal{R}}_{0}\right],
$$

where the diagonal terms of $\hat{I}^{(1)}, \mathcal{I}_{\mu}^{a, c}\left[\hat{\mathcal{R}}_{0}\right]$, depend only on the diagonal elements of $\hat{\mathcal{R}}_{0}(\mathbf{p}), \hat{\mathcal{R}}_{0 \mu \mu}(\mathbf{p})$, and are given by Eqs. (A.1) and A.3). Since $\mathcal{I}_{\mu}^{c}\left[\hat{\mathcal{R}}_{0}\right]$ is a higher-order term in the electron-impurity scattering, we can assume that $\mathcal{I}_{\mu}^{c}\left[\hat{\mathcal{R}}_{0}\right]$ is much smaller than $\mathcal{I}_{\mu}^{a}\left[\hat{\mathcal{R}}_{0}\right]$. Hence, Eq. (30) can be solved as follows: we first solve Eq. (30) by ignoring $\mathcal{I}_{\mu}^{c}\left[\hat{\mathcal{R}}_{0}\right]$ and then substitute the obtained solution into $\mathcal{I}_{\mu}^{c}$ to calculate a correction caused by $\mathcal{I}_{\mu}^{c}$. Thus, we find that the solution of Eq. (30) consists of two terms: $\hat{\mathcal{R}}_{0 \mu \mu}(\mathbf{p})=\hat{\mathcal{R}}_{0 \mu \mu}^{s}(\mathbf{p})+\hat{\mathcal{R}}_{0 \mu \mu}^{c}(\mathbf{p})$, with $\hat{\mathcal{R}}_{0 \mu \mu}^{s}(\mathbf{p})$ and $\hat{\mathcal{R}}_{0 \mu \mu}^{c}(\mathbf{p})$, respectively, determined by

$$
e \mathbf{E} \cdot \nabla_{\mathbf{p}} \hat{\rho}_{0 \mu \mu}(\mathbf{p})=-\mathcal{I}_{\mu}^{a}\left[\hat{\mathcal{R}}_{0}^{s}\right]
$$

and

$$
\mathcal{I}_{\mu}^{a}\left[\hat{\mathcal{R}}_{0}^{c}\right]+\mathcal{I}_{\mu}^{c}\left[\hat{\mathcal{R}}_{0}^{s}\right]=0
$$

The solution of Eq. (31) $\hat{\mathcal{R}}_{0 \mu \mu}^{s}(\mathbf{p})$, depends on the momentum angle through $\sin \phi_{\mathbf{p}}$ and takes the form, $\hat{\mathcal{R}}_{0 \mu \mu}^{s}(\mathbf{p})=$ $e E \Phi_{0 \mu}^{s}(p) \sin \phi_{\mathbf{p}}$, with the functions $\Phi_{0 \mu}^{s}(p)$ given by

$$
\Phi_{0 \mu}^{s}(p)=-\frac{\partial n_{\mathrm{F}}\left(\varepsilon_{\mu}(p)\right)}{\partial \varepsilon_{\mu}(p)} \frac{\left(\tau_{1 \bar{\mu} \bar{\mu}}^{-1}+\tau_{2 \bar{\mu} \mu}^{-1}\right) \frac{\partial \varepsilon_{\mu}(p)}{\partial p}+\tau_{3 \mu \bar{\mu}}^{-1} \frac{\partial \varepsilon_{\bar{\mu}}\left(\tilde{p}_{\mu}\right)}{\partial \tilde{p}_{\mu}}}{\left(\tau_{1 \bar{\mu} \bar{\mu}}^{-1}+\tau_{2 \bar{\mu} \mu}^{-1}\right)\left(\tau_{1 \mu \mu}^{-1}+\tau_{2 \mu \bar{\mu}}^{-1}\right)-\tau_{3 \bar{\mu} \mu}^{-1} \tau_{3 \mu \bar{\mu}}^{-1}},
$$

where $\tilde{p}_{\mu}$ is given by equation $\varepsilon_{\bar{\mu}}\left(\tilde{p}_{\mu}\right)=\varepsilon_{\mu}(p)$ and the various relaxation times $\tau_{i \mu \nu}(i=1 . .3, \mu, \nu=1,2)$ are defined as

$$
\frac{1}{\tau_{i \mu \nu}}=2 \pi n_{i} \sum_{\mathbf{k}}|V(\mathbf{p}-\mathbf{k})|^{2} \Lambda_{i \mu \nu}(\mathbf{p}, \mathbf{k})
$$

with $\Lambda_{1 \mu \nu}(\mathbf{p}, \mathbf{k})=\frac{1}{2}\left[1-\cos \left(\phi_{\mathbf{p}}-\phi_{\mathbf{k}}\right)\right] a_{+}(\mathbf{p}, \mathbf{k}) \Delta_{\mu \nu}, \Lambda_{2 \mu \nu}(\mathbf{p}, \mathbf{k})=\frac{1}{2} a_{-}(\mathbf{p}, \mathbf{k}) \Delta_{\mu \nu}, \Lambda_{3 \mu \nu}(\mathbf{p}-\mathbf{k})=\frac{1}{2} \cos \left(\phi_{\mathbf{p}}-\right.$ $\left.\phi_{\mathbf{k}}\right) a_{-}(\mathbf{p}, \mathbf{k}) \Delta_{\mu \nu}$ and $a_{ \pm}(\mathbf{p}, \mathbf{k}) \equiv\left(\lambda_{p} \lambda_{k} \pm M^{2} \pm \alpha^{2} k p \cos \left(\phi_{\mathbf{p}}-\phi_{\mathbf{k}}\right)\right) / \lambda_{p} \lambda_{k}$. Substituting the result for $\hat{\mathcal{R}}_{0 \mu \mu}^{s}(\mathbf{p})$ into $\mathcal{I}_{\mu}^{c}$, we find that the solution of Eq. (32), $\hat{\mathcal{R}}_{0 \mu \mu}^{c}(\mathbf{p})$, relates to a cosine function of the momentum angle: $\hat{\mathcal{R}}_{0 \mu \mu}(\mathbf{p})=e E \Phi_{0 \mu}^{c}(p) \cos \phi_{\mathbf{p}} . \Phi_{0 \mu}^{c}(p)$ is given by

$$
\Phi_{0 \mu}^{c}(p)=-\frac{\left(\tau_{1 \bar{\mu} \bar{\mu}}^{-1}+\tau_{2 \bar{\mu} \mu}^{-1}\right) \mathcal{L}_{0 \mu}^{c}(p)+\tau_{3 \mu \bar{\mu}}^{-1} \mathcal{L}_{0 \bar{\mu}}^{c}\left(\tilde{p}_{\mu}\right)}{\left(\tau_{1 \bar{\mu} \bar{\mu}}^{-1}+\tau_{2 \bar{\mu} \mu}^{-1}\right)\left(\tau_{1 \mu \mu}^{-1}+\tau_{2 \mu \bar{\mu}}^{-1}\right)-\tau_{3 \bar{\mu} \mu}^{-1} \tau_{3 \mu \bar{\mu}}^{-1}},
$$

with

$$
\begin{aligned}
\mathcal{L}_{0 \mu}^{c}(p)= & \pi^{2} \alpha^{2} M n_{i} \sum_{\mathbf{k}, \mathbf{q}} V_{\mathbf{p}-\mathbf{k}} V_{\mathbf{k}-\mathbf{q}} V_{\mathbf{q}-\mathbf{p}} \frac{1}{\lambda_{p} \lambda_{k}} \sin \left(\phi_{\mathbf{k}}-\phi_{\mathbf{p}}\right) \\
& \times\left\{c_{\mu+}(\mathbf{p}, \mathbf{q}, \mathbf{k}) \Delta_{\mu \mu} \Phi_{0 \mu}^{s}(p)+c_{\mu-}(\mathbf{p}, \mathbf{q}, \mathbf{k}) \Delta_{\mu \bar{\mu}} \Phi_{0 \bar{\mu}}^{s}\left(\tilde{p}_{\mu}\right)\right\},
\end{aligned}
$$

and $c_{\mu \pm}(\mathbf{p}, \mathbf{q}, \mathbf{k})$ defined by Eq. A.5.

The off-diagonal $\hat{\mathcal{R}}_{0}(\mathbf{p})$ elements, $\hat{\mathcal{R}}_{0 \mu \bar{\mu}}(\mathbf{p})$, are obtained by substituting $\hat{\mathcal{R}}_{0 \mu \mu}^{s}(\mathbf{p})$ into the off-diagonal parts of Eq. (26):

$$
i\left[\hat{H}_{0}, \hat{\mathcal{R}}_{0}(\mathbf{p})\right]=-\mathcal{S}^{a}\left[\hat{\mathcal{R}}_{0}^{s}\right] .
$$

Here, the effect of $\hat{\mathcal{R}}_{0 \mu \mu}^{c}(\mathbf{p})$ on the off-diagonal elements was ignored. We find that $\hat{\mathcal{R}}_{0 \mu \bar{\mu}}(\mathbf{p})$ depends on the momentum angle not only through $\sin \phi_{\mathbf{p}}$ but also through $\cos \phi_{\mathbf{p}}$. However, as discussed above, we are interested only in the part of $\operatorname{Re}\left[\left(\hat{\mathcal{R}}_{0}\right)_{12}(\mathbf{p})\right]$, which depends on $\phi_{\mathbf{p}}$ through $\cos \phi_{\mathbf{p}}$, and the part of $\operatorname{Im}\left[\left(\hat{\mathcal{R}}_{0}\right)_{12}(\mathbf{p})\right]$, involving $\sin \phi_{\mathbf{p}}$ : $\operatorname{Re}\left[\left(\hat{\mathcal{R}}_{0}\right)_{12}(\mathbf{p})\right]=\xi_{0}^{I}(p) \cos \phi_{\mathbf{p}}+\ldots$ and $\operatorname{Im}\left[\left(\hat{\mathcal{R}}_{0}\right)_{12}(\mathbf{p})\right]=\zeta_{0}^{I}(p) \sin \phi_{\mathbf{p}}+\ldots$, with $\xi_{0}^{I}(p)$ and $\zeta_{0}^{I}(p)$ taking the forms,

$$
\begin{aligned}
\zeta_{0}^{I}(p)= & \frac{e E \pi n_{i}}{4 \lambda_{p}} \sum_{\mathbf{k} \mu=1,2}|V(\mathbf{p}-\mathbf{k})|^{2} \operatorname{Im}\left[g_{a}(\mathbf{p}, \mathbf{k})\right](-1)^{\mu}\left\{\Delta_{\mu \mu} \Phi_{0 \mu}^{s}(p)\left[1-\cos \left(\phi_{\mathbf{p}}-\phi_{\mathbf{k}}\right)\right]\right. \\
& \left.-\Delta_{\mu \bar{\mu}}\left[\Phi_{0 \mu}^{s}(p)-\Phi_{0 \bar{\mu}}^{s}\left(\tilde{p}_{\mu}\right) \cos \left(\phi_{\mathbf{p}}-\phi_{\mathbf{k}}\right)\right]\right\},
\end{aligned}
$$


and

$$
\begin{aligned}
\xi_{0}^{I}(p)= & \frac{e E \pi n_{i}}{4 \lambda_{p}} \sum_{\mathbf{k} \mu=1,2}|V(\mathbf{p}-\mathbf{k})|^{2} \operatorname{Re}\left[g_{a}(\mathbf{p}, \mathbf{k})\right](-1)^{\mu} \\
& \times\left\{\Delta_{\mu \mu} \Phi_{0 \mu}^{s}(p) \sin \left(\phi_{\mathbf{p}}-\phi_{\mathbf{k}}\right)-\Delta_{\mu \bar{\mu}} \Phi_{0 \bar{\mu}}^{s}\left(\tilde{p}_{\mu}\right) \sin \left(\phi_{\mathbf{p}}-\phi_{\mathbf{k}}\right)\right\}
\end{aligned}
$$

and $g_{a}(\mathbf{p}, \mathbf{k})$ defined as $g_{a}(\mathbf{p}, \mathbf{k}) \equiv\left[\alpha k \lambda_{p} \sin \left(\phi_{\mathbf{p}}-\phi_{\mathbf{k}}\right)+i \alpha M\left(p-k \cos \left(\phi_{\mathbf{p}}-\phi_{\mathbf{k}}\right)\right)\right] /\left(\lambda_{k} \lambda_{p}\right)$.

To obtain the first-order term of the distribution function in the $\lambda$ expansion, $\hat{\mathcal{R}}_{1}(\mathbf{p})$, we substitute $\hat{\mathcal{R}}_{0 \mu \mu}^{s}(\mathbf{p})$ into the diagonal components of the scattering term, $\mathcal{I}_{\mu}^{b}$ and $\mathcal{I}_{\mu}^{d}$, as well as its off-diagonal component $\mathcal{S}^{b}$. We find that $\mathcal{I}_{\mu}^{b}\left[\hat{\mathcal{R}}_{0}^{s}\right]$ depends on the angle of momentum through a sine function, while $\mathcal{I}_{\mu}^{d}\left[\hat{\mathcal{R}}_{0}^{s}\right]$ relates to a cosine function: $\mathcal{I}_{\mu}^{b}\left[\hat{\mathcal{R}}_{0}^{s}\right]=\mathcal{L}_{1 \mu}^{s}(p) \sin \phi_{\mathbf{p}}$ and $\mathcal{I}_{1 \mu}^{d}\left[\hat{\mathcal{R}}_{0}^{s}\right]=\mathcal{L}_{1 \mu}^{c}(p) \cos \phi_{\mathbf{p}}$ with $\mathcal{L}_{1 \mu}^{c, s}$ given by

$$
\begin{aligned}
\mathcal{L}_{1 \mu}^{s}(p)= & 2 \pi n_{i} \lambda \alpha^{2} \sum_{\mathbf{k}}|V(\mathbf{p}-\mathbf{k})|^{2} \frac{k^{2} p^{2}}{\lambda_{p} \lambda_{k}} \sin ^{2}\left(\phi_{\mathbf{k}}-\phi_{\mathbf{p}}\right) \\
& \times\left\{\left[1-\cos \left(\phi_{\mathbf{k}}-\phi_{\mathbf{p}}\right)\right] \Phi_{0 \mu}^{s}(p) \Delta_{\mu \mu}-\left[\Phi_{0 \mu}^{s}(p)-\Phi_{0 \bar{\mu}}^{s}\left(\tilde{p}_{\mu}\right) \cos \left(\phi_{\mathbf{k}}-\phi_{\mathbf{p}}\right)\right] \Delta_{\mu \bar{\mu}}\right\}
\end{aligned}
$$

and

$$
\begin{aligned}
\mathcal{L}_{1 \mu}^{c}(p)= & \pi^{2} \lambda M n_{i} \sum_{\mathbf{k}, \mathbf{q}} V_{\mathbf{p}-\mathbf{k}} V_{\mathbf{k}-\mathbf{q}} V_{\mathbf{q}-\mathbf{p}} \frac{1}{\lambda_{p} \lambda_{k}} \sin \left(\phi_{\mathbf{k}}-\phi_{\mathbf{p}}\right) \\
& \times\left\{d_{\mu+}(\mathbf{p}, \mathbf{q}, \mathbf{k}) \Delta_{\mu \mu} \Phi_{0 \mu}^{s}(p)+d_{\mu-}(\mathbf{p}, \mathbf{q}, \mathbf{k}) \Delta_{\mu \bar{\mu}} \Phi_{0 \bar{\mu}}^{s}\left(\tilde{p}_{\mu}\right)\right\} .
\end{aligned}
$$

Here, $d_{\mu \pm}(\mathbf{p}, \mathbf{q}, \mathbf{k})$ are defined by Eq. (A.6). From the diagonal parts of Eq. (26) in the first order of $\lambda, \mathcal{I}_{\mu}^{a}\left[\hat{\mathcal{R}}_{1}\right]+$ $\mathcal{I}_{\mu}^{b}\left[\hat{\mathcal{R}}_{0}^{s}\right]+\mathcal{I}_{\mu}^{d}\left[\hat{\mathcal{R}}_{0}^{s}\right]=0$, it follows that $\hat{\mathcal{R}}_{1 \mu \mu}(\mathbf{p})$ can be written as

$$
\hat{\mathcal{R}}_{1 \mu \mu}(\mathbf{p})=e E \Phi_{1 \mu}^{s}(p) \sin \phi_{\mathbf{p}}+e E \Phi_{1 \mu}^{c}(p) \cos \phi_{\mathbf{p}}
$$

with $\Phi_{1 \mu}^{s, c}(p)$ determined by

$$
\Phi_{1 \mu}^{s, c}(p)=-\frac{\left(\tau_{1 \bar{\mu} \bar{\mu}}^{-1}+\tau_{2 \bar{\mu} \mu}^{-1}\right) \mathcal{L}_{1 \mu}^{s, c}(p)+\tau_{3 \mu \bar{\mu}}^{-1} \mathcal{L}_{1 \bar{\mu}}^{s, c}\left(\tilde{p}_{\mu}\right)}{\left(\tau_{1 \bar{\mu} \bar{\mu}}^{-1}+\tau_{2 \bar{\mu} \mu}^{-1}\right)\left(\tau_{1 \mu \mu}^{-1}+\tau_{2 \mu \bar{\mu}}^{-1}\right)-\tau_{3 \bar{\mu} \mu}^{-1} \tau_{3 \mu \bar{\mu}}^{-1}} .
$$

The off-diagonal elements of $\hat{\mathcal{R}}_{1}(\mathbf{p})$ are obtained from the off-diagonal parts of Eq. (26) in the first order of $\lambda$ : $i\left[\hat{H}_{0}, \hat{\mathcal{R}}_{1}(\mathbf{p})\right]=-\mathcal{S}^{b}\left[\hat{\mathcal{R}}_{0}^{s}\right]$. According to the definitions, $\operatorname{Re}\left[\left(\hat{\mathcal{R}}_{1}\right)_{12}(\mathbf{p})\right]=\xi_{1}^{I}(p) \cos \phi_{\mathbf{p}}+\ldots$ and $\operatorname{Im}\left[\left(\hat{\mathcal{R}}_{1}\right)_{12}(\mathbf{p})\right]=$ $\zeta_{1}^{I}(p) \sin \phi_{\mathbf{p}}+\ldots, \xi_{1}^{I}(p)$ and $\zeta_{1}^{I}(p)$ can be written as

$$
\begin{aligned}
\zeta_{1}^{I}(p)= & \frac{e E \pi n_{i}}{4 \lambda_{p}} \sum_{\mathbf{k} \mu=1,2}|V(\mathbf{p}-\mathbf{k})|^{2} \operatorname{Im}\left[g_{b}(\mathbf{p}, \mathbf{k})\right](-1)^{\mu}\left\{\Delta_{\mu \mu} \Phi_{0 \mu}^{s}(p)\left[1-\cos \left(\phi_{\mathbf{p}}-\phi_{\mathbf{k}}\right)\right]\right. \\
& \left.-\Delta_{\mu \bar{\mu}}\left[\Phi_{0 \mu}^{s}(p)-\Phi_{0 \bar{\mu}}^{s}\left(\tilde{p}_{\mu}\right) \cos \left(\phi_{\mathbf{p}}-\phi_{\mathbf{k}}\right)\right]\right\}
\end{aligned}
$$

and

$$
\xi_{1}^{I}(p)=\frac{e E \pi n_{i}}{4 \lambda_{p}} \sum_{\mathbf{k} \mu=1,2}|V(\mathbf{p}-\mathbf{k})|^{2} \operatorname{Re}\left[g_{b}(\mathbf{p}, \mathbf{k})\right](-1)^{\mu} \sin \left(\phi_{\mathbf{p}}-\phi_{\mathbf{k}}\right)\left\{\Delta_{\mu \mu} \Phi_{0 \mu}^{s}(p)-\Delta_{\mu \bar{\mu}} \Phi_{0 \bar{\mu}}^{s}\left(\tilde{p}_{\mu}\right)\right\} .
$$

The other component of the kinetic equation, Eq. (27), can be solved easily. The solution $\hat{\rho}_{1}^{I I}(\mathbf{p})$ has null diagonal elements. Its off-diagonal elements can be written as $\left(\rho_{1}^{I I}\right)_{12}(\mathbf{p})=\xi^{I I}(p) \cos \phi_{\mathbf{p}}+i \zeta^{I I}(p) \sin \phi_{\mathbf{p}}$ with $\xi^{I I}(p)$ and $\zeta^{I I}(p)$ defined as

$$
\xi^{I I}(p)=\frac{\alpha e E}{4 \lambda_{p}^{2}}\left(1-2 \lambda p^{2}\right)\left\{n_{\mathrm{F}}\left[\varepsilon_{1}(p)\right]-n_{\mathrm{F}}\left[\varepsilon_{2}(p)\right]\right\}
$$

and

$$
\zeta^{I I}(p)=\frac{\alpha e E M}{4 \lambda_{p}^{3}}\left\{n_{\mathrm{F}}\left[\varepsilon_{1}(p)\right]-n_{\mathrm{F}}\left[\varepsilon_{2}(p)\right]\right\}
$$




\section{Anomalous Hall current}

We first analyze the component of the anomalous Hall current, $J_{x}^{\mathrm{f}}$, that is associated with the current operator term arising from the free-electron Hamiltonian and is a sum of contributions from the diagonal and off-diagonal elements of the distribution function: $J_{x}^{\mathrm{f}}=J_{x}^{\mathrm{fd}}+J_{x}^{\mathrm{fo}}$. We know that $J_{x}^{\mathrm{fd}}$ is a component of the skew-scattering AHC. Considering Eq. (13), it is obvious that the nonvanishing $J_{x}^{\mathrm{fd}}$ comes from the diagonal terms of the distribution function, $\hat{\mathcal{R}}_{0 \mu \mu}^{c}(\mathbf{p})$ and $\hat{\mathcal{R}}_{1 \mu \mu}^{c}(\mathbf{p})$, which depend on momentum angle through the cosine function. Thus, $J_{x}^{\mathrm{fd}}$ can be written as $J_{x}^{\mathrm{fd}}=J_{x}^{\mathrm{ss}-\mathrm{L}}+J_{x}^{\mathrm{ss}-\mathrm{F}}$, where $J_{x}^{\mathrm{ss}-\mathrm{L}}$ and $J_{x}^{\mathrm{ss}-\mathrm{F}}$, respectively, are associated with the lowest- and first-order terms of the distribution function, $\hat{\mathcal{R}}_{0 \mu \mu}^{c}(\mathbf{p})$ and $\hat{\mathcal{R}}_{1 \mu \mu}^{c}(\mathbf{p})$ :

$$
\begin{aligned}
& J_{x}^{\mathrm{ss}-\mathrm{L}}=e^{2} E \sum_{\mathbf{p}, \mu}\left\{\left(\frac{1}{m^{*}}+(-1)^{\mu} \frac{\alpha^{2}}{\lambda_{p}}\right) p \cos ^{2} \phi_{\mathbf{p}} \Phi_{0 \mu}^{c}(p)\right\}, \\
& J_{x}^{\mathrm{ss}-\mathrm{F}}=e^{2} E \sum_{\mathbf{p}, \mu}\left\{\left(\frac{1}{m^{*}}+(-1)^{\mu} \frac{\alpha^{2}}{\lambda_{p}}\right) p \cos ^{2} \phi_{\mathbf{p}} \Phi_{1 \mu}^{c}(p)\right\} .
\end{aligned}
$$

Note that both $J_{x}^{\mathrm{ss}-\mathrm{L}}$ and $J_{x}^{\mathrm{ss}-\mathrm{F}}$ are proportional to the inverse of the impurity-density, i.e. $\left(n_{i}\right)^{-1}$, appearing when electron-impurity scattering is considered up to the second-Born approximation.

Since both the distribution terms $\hat{\rho}^{I}(\mathbf{p})$ and $\hat{\rho}^{I I}(\mathbf{p})$ have nonvanishing off-diagonal elements, the contribution to the anomalous Hall current from off-diagonal elements of $\hat{\rho}(\mathbf{p}), J_{x}^{\mathrm{fo}}$, can be expressed as $J_{x}^{\mathrm{fo}}=\left.J_{x}^{\mathrm{fo}}\right|^{I}+\left.J_{x}^{\mathrm{fo}}\right|^{I I}$, where $\left.J_{x}^{\mathrm{fo}}\right|^{I}$ and $\left.J_{x}^{\mathrm{fo}}\right|^{I I}$ arise from $\hat{\rho}_{12}^{I}(\mathbf{p})$ and $\hat{\rho}_{12}^{I I}(\mathbf{p})$, respectively, and take the forms

$$
\left.J_{x}^{\mathrm{fo}}\right|^{I}=2 e^{2} E \sum_{\mathbf{p}}\left\{\frac{\alpha M}{\lambda_{p}}\left[\xi_{0}^{I}(p)+\xi_{1}^{I}(p)\right] \cos ^{2} \phi_{\mathbf{p}}+\alpha\left[\zeta_{0}^{I}(p)+\zeta_{1}^{I}(p)\right] \sin ^{2} \phi_{\mathbf{p}}\right\},
$$

and

$$
\begin{aligned}
\left.J_{x}^{\mathrm{fo}}\right|^{I I} & =2 e^{2} E \sum_{\mathbf{p}}\left\{\frac{\alpha M}{\lambda_{p}} \xi^{I I}(p) \cos ^{2} \phi_{\mathbf{p}}+\alpha \zeta^{I I}(p) \sin ^{2} \phi_{\mathbf{p}}\right\} \\
& =\frac{M \alpha^{2} e^{2} E}{2} \sum_{\mathbf{p}} \frac{1}{\lambda_{p}^{3}}\left(1-\lambda p^{2}\right)\left\{n_{\mathrm{F}}\left[\varepsilon_{1}(p)\right]-n_{\mathrm{F}}\left[\varepsilon_{2}(p)\right]\right\} .
\end{aligned}
$$

From Eq. (501) we see that $\left.J_{x}^{\mathrm{fo}}\right|^{I}$ is independent of the impurity density, due to the $n_{i}$-independence of $\xi_{0}^{I}$ and $\zeta_{1}^{I}$. However, it is due to disorder and relates to longitudinal transport. It is obvious that $\left.J_{x}^{\text {fo }}\right|^{I}$ involves the derivative of the equilibrium distribution function, i.e. $\partial n_{\mathrm{F}}(\omega) / \partial \omega$. This implies that $\left.J_{x}^{\mathrm{fo}}\right|^{I}$ arises only from electron states in the vicinity of the Fermi surface, or, in other words, from electron states involved in longitudinal transport. Physically, the electrons participating in transport experience impurity scattering, producing diagonal $\hat{\rho}_{1}^{I}(\mathbf{p})$ elements of order of $n_{i}^{-1}$. Moreover, the scattering of these perturbed electrons by impurities also gives rise to an interband polarization, which eliminates dependence on the impurity density within the diffusive regime.

However, the anomalous Hall current $\left.J_{x}^{\mathrm{fo}}\right|^{I I}$ is a function of the entire unperturbed equilibrium distribution, $n_{\mathrm{F}}(\omega)$, not just of its derivative, $\partial n_{\mathrm{F}}(\omega) / \partial \omega$, at the Fermi surface. This indicates that $\left.J_{x}^{\text {fo }}\right|^{I I}$ has contributions from all electron states below the Fermi sea. Obviously, $\left.J_{x}^{\mathrm{fo}}\right|^{I I}$ is independent of any electron-impurity scattering and relates to the driving terms, one of which is just the interband electric dipole moment, while the other one arises from the SO coupling directly induced by the driving electric field.

From Eq. (10), it is obvious that to determine the first-order term of $J_{x}^{\text {imp }}$ in the $\lambda$-power expansion, one has to deal with the function $<\check{\psi}_{\nu \mathbf{p}}^{+} \breve{\psi}_{\mu \mathbf{k}}>$ in the lowest order of $\lambda$. We find that this lowest-order term of $<\check{\psi}_{\nu \mathbf{p}}^{+} \check{\psi}_{\mu \mathbf{k}}>$ can be evaluated from the kinetic equation for the distribution function $\check{\rho}(\mathbf{p})$. To show this, we start with a Heisenberg equation for the operator $\check{\psi}_{\nu \mathbf{p}}^{+} \check{\psi}_{\mu \mathbf{p}}$ :

$$
i \hbar \frac{\partial}{\partial T} \check{\psi}_{\nu \mathbf{p}}^{+} \check{\psi}_{\mu \mathbf{p}}=\left[\check{H}, \check{\psi}_{\nu \mathbf{p}}^{+} \check{\psi}_{\mu \mathbf{p}}\right]=-i e \mathbf{E} \cdot \nabla_{\mathbf{p}}\left[\check{\psi}_{\nu \mathbf{p}}^{+} \check{\psi}_{\mu \mathbf{p}}\right]+\left[\check{H}_{0}+\check{H}_{E}, \check{\psi}_{\nu \mathbf{p}}^{+} \check{\psi}_{\mu \mathbf{p}}\right]+\check{I}_{\mu \nu}^{s}(\mathbf{p}),
$$

where, $\check{I}_{\mu \nu}^{s}(\mathbf{p}) \equiv\left[\check{H}_{\mathrm{imp}}, \check{\psi}_{\nu \mathbf{p}}^{+} \check{\psi}_{\mu \mathbf{p}}\right]$. In the lowest order of $\lambda, \check{I}_{\mu \nu}^{s}(\mathbf{p})$ takes the form,

$$
\check{I}_{\mu \nu}^{s}(\mathbf{p}) \approx \sum_{\mathbf{k}, i} V_{\mathbf{p}-\mathbf{k}}\left[\mathrm{e}^{i \mathbf{R}_{i} \cdot(\mathbf{p}-\mathbf{k})} \check{\psi}_{\nu \mathbf{k}}^{+} \check{\psi}_{\mu \mathbf{p}}-\mathrm{e}^{i \mathbf{R}_{i} \cdot(\mathbf{k}-\mathbf{p})} \check{\psi}_{\nu \mathbf{p}}^{+} \check{\psi}_{\mu \mathbf{k}}\right] .
$$


Multiplying both sides of Eq. (52) by $\varepsilon_{l m n} p_{m} \sigma_{\nu \mu}^{n}$ and taking the summation over $\mu$ and $\nu$, we get

$$
\sum_{\mathbf{p}, \mu \nu} \varepsilon_{l m n}\left[p_{m} \sigma_{\nu \mu}^{n}<\check{I}_{\mu \nu}^{s}(\mathbf{p})>\right] \approx \sum_{\mathbf{p}, \mathbf{k}, \mu \nu} \mathrm{e}^{i \mathbf{R}_{i} \cdot(\mathbf{k}-\mathbf{p})} V_{\mathbf{k}-\mathbf{p}} \varepsilon_{l m n}\left(k_{m}-p_{m}\right)\left[<\check{\psi}_{\nu \mathbf{p}}^{+} \check{\psi}_{\mu \mathbf{k}}>\sigma_{\nu \mu}^{n}\right],
$$

with $\langle\ldots\rangle$ denoting a statistical average. Obviously, the right-hand side of Eq. (54) is just the AHC component $J_{l}^{\mathrm{imp}}$. On the other hand, taking the statistical average of Eq. (52) reduces it to the kinetic equation for $\check{\rho}_{\mu \nu}(\mathbf{p})=<\check{\psi}_{\nu \mathbf{p}}^{+} \check{\psi}_{\mu \mathbf{p}}>$. Hence, from Eqs. (52) and (54), it follows that $J_{l}^{\mathrm{imp}}$ can be written in the spin basis as

$$
J_{l}^{\mathrm{imp}}=-\lambda e \sum_{\mathbf{p}} \varepsilon_{l m n} p_{m} \operatorname{Tr}\left\{\sigma^{n}\left(e \mathbf{E} \cdot \nabla_{\mathbf{p}} \check{\rho}(\mathbf{p})+i\left[\check{H}_{0}, \check{\rho}(\mathbf{p})\right]\right)\right\},
$$

where the contribution associated with $\check{H}_{E}$ is ignored because it is of higher order in $\lambda . J_{l}^{\text {imp }}$ can also be determined in the helicity basis by means of

$$
J_{l}^{\mathrm{imp}}=-\lambda e \sum_{\mathbf{p}} \varepsilon_{l m n} p_{m} \operatorname{Tr}\left\{U_{\mathbf{p}}^{+} \sigma^{n} U_{\mathbf{p}}\left[e \mathbf{E} \cdot \nabla_{\mathbf{p}} \hat{\rho}(\mathbf{p})-e \mathbf{E} \cdot\left[\hat{\rho}(\mathbf{p}), U_{\mathbf{p}}^{+} \nabla_{\mathbf{p}} U_{\mathbf{p}}\right]+i\left[\hat{H}_{0}, \hat{\rho}(\mathbf{p})\right]\right]\right\} .
$$

In the linear response regime, Eq. (56) reduces to

$$
\begin{aligned}
J_{x}^{\mathrm{imp}}= & -\lambda e \sum_{\mathbf{p}} \frac{p_{y}}{\lambda_{p}}\left\{e E M \nabla_{p_{y}}\left[\left(\hat{\rho}_{0}\right)_{11}(\mathbf{p})-\left(\hat{\rho}_{0}\right)_{22}(\mathbf{p})\right]\right. \\
& \left.-\frac{e E \alpha^{2} p}{\lambda_{p}^{2}} M \sin \phi_{\mathbf{p}}\left[\left(\hat{\rho}_{0}\right)_{11}(\mathbf{p})-\left(\hat{\rho}_{0}\right)_{22}(\mathbf{p})\right]+4 \alpha p \lambda_{p}\left[\zeta_{0}^{I}(p)+\zeta^{I I}(p)\right] \sin \phi_{\mathbf{p}}\right\} \\
= & -\lambda e \sum_{\mathbf{p}}\left\{-\frac{e E M}{\lambda_{p}}\left\{n_{F}\left[\varepsilon_{1}(p)\right]-n_{F}\left[\varepsilon_{2}(p)\right]\right\}+4 \alpha p p_{y}\left[\zeta_{0}^{I}(p)+\zeta^{I I}(p)\right] \sin \phi_{\mathbf{p}}\right\},
\end{aligned}
$$

To obtain the last equality in Eq. (57), the momentum integral with integrand involving the derivative with respect to $p_{y}$ was performed by parts. Note that Sinitsyn, et al. recently investigated this component of the anomalous Hall current in the absence of Rashba SO coupling by analyzing the effect of impurity scattering on the coordinate shift of the electron wave-packet 15

$J_{x}^{E}$ arises from SO coupling directly induced by the driving electric field. Considered to linear order in the electric field, it takes the form

$$
J_{x}^{E}=\lambda e^{2} E \sum_{\mathbf{p}}\left\{n_{F}\left[\varepsilon_{1}(p)\right]-n_{F}\left[\varepsilon_{2}(p)\right]\right\}
$$

Obviously, this contribution to the anomalous Hall current is independent of any electron-impurity scattering. If only one of the parameters-the Rashba SO coupling constant or the magnetization-is zero and other remains finite, $J_{x}^{E}$ doesn't vanish. In contrast to this, $J_{x}^{\mathrm{fd}}$ and $J_{x}^{\mathrm{fo}}$, as well as $J_{x}^{\mathrm{imp}}$ reduce to zero for just one of them vanishing, null $\alpha$ or $M$. Note that in all previous studies, the contribution to anomalous Hall current from the SO term due to the driving electric field has been ignored. In the following numerical calculation, we will show that $J_{x}^{E}$ plays an important role, especially in the low magnetization regime.

Thus, after all components of AHC are determined, the total anomalous Hall current can be obtained from Eq. (9). We define the total anomalous Hall conductivity as $\sigma_{x y}=J_{x} / E$. Obviously, $\sigma_{x y}$ can be written as

$$
\sigma_{x y}=\sigma_{x y}^{\mathrm{sj}}+\sigma_{x y}^{\mathrm{ss}},
$$

with $\sigma_{x y}^{\mathrm{sj}}=J_{x}^{\mathrm{sj}} / E=\sigma_{x y}^{\mathrm{fo}}+\sigma_{x y}^{\mathrm{imp}}+\sigma_{x y}^{E}$ and $\sigma_{x y}^{\mathrm{ss}}=J_{x}^{\mathrm{ss}} / E=\sigma_{x y}^{\mathrm{ss}-\mathrm{L}}+\sigma_{x y}^{\mathrm{ss}-\mathrm{F}}$. Here, the quantities, $\sigma_{x y}^{\mathrm{fo}}, \sigma_{x y}^{\mathrm{imp}}, \sigma_{x y}^{E}, \sigma_{x y}^{\mathrm{ss}-\mathrm{L}}$, and $\sigma_{x y}^{\mathrm{ss}-\mathrm{L}}$, are defined as $\sigma_{x y}^{\mathrm{imp}, E}=J_{x}^{\mathrm{imp}, E} / E, \sigma_{x y}^{\mathrm{fo}}=J_{x}^{\mathrm{fo}} / E, \sigma_{x y}^{\mathrm{ss}-\mathrm{L}}=J_{x}^{\mathrm{ss}-\mathrm{L}} / E$ and $\sigma_{x y}^{\mathrm{ss}-\mathrm{F}}=J_{x}^{\mathrm{ss}-\mathrm{F}} / E$.

It should be noted that, in our study, the diagonal part of the distribution function, $\hat{\mathcal{R}}_{0}^{s}(\mathbf{p})$, which is involved in all disorder-related components of anomalous Hall current, was evaluated in the self-consistent Born approximation. This implies that our results correspond to that obtained in the Kubo formalism by considering the "ladder-sum" vertex corrections to the bubble diagrams. 


\section{RESULTS AND DISCUSSIONS}

We have carried out a numerical calculation to investigate the anomalous Hall effect in a InSb/AlInSb quantum well with Rashba SO coupling. Such a system was recently examined experimentally ${ }^{32}$ It is well known that the InSb semiconductor is a good material for AHE observation because its band gap, $E_{0}=0.235 \mathrm{eV}$, spin-orbit splitting, $\Delta_{\mathrm{SO}}=0.81 \mathrm{eV}$, and $P=9.63 \mathrm{eV} \cdot \AA$ result in a pronounced spin-orbit coupling constant $\lambda=5.31 \mathrm{~nm}^{2}($ for $\mathrm{GaAs}$, $\left.\lambda=0.053 \mathrm{~nm}^{2}\right)^{33}$ Also, the large g-factor, $g=-51.4$, may lead to a remarkably large magnetization. In our calculation, the static dielectric constant, $\kappa$, and the effective mass of InSb, $m^{*}$, are chosen to be $\kappa=17.54$ and $m^{*}=0.0135 m_{0}$ with $m_{0}$ as the free electron mass. The width of the InSb/AlInSb quantum well is assumed to be $a=20 \mathrm{~nm}$ and the density of electrons is taken as $N_{e}=1 \times 10^{15} \mathrm{~m}^{-2}$. We consider an attractive interaction between the electrons and the background impurities in the quantum wells (the attractive and repulsive interactions lead to differing anomalous Hall effects because their contributions to AHC in the second Born approximation have opposite signs). Note that we have also estimated the effect of scattering of electrons by remote impurities on AHE, finding that it is relatively small and can be ignored. Thus, the scattering potential $V_{\mathbf{q}}$ can be written as 34

$$
V_{\mathbf{q}}=U(q) F(q) / \kappa(q, 0),
$$

with $U(q)=-e^{2} /\left(2 \varepsilon_{0} \kappa q\right)$ and the form factor $F(q)$ determined by $(u=q a)$

$$
F(q)=\frac{8 \pi^{2}}{\left(4 \pi^{2}+u^{2}\right) u}\left[1+\frac{u^{2}}{4 \pi^{2}}-\frac{1-\exp (-u)}{u}\right] .
$$

$\kappa(q, 0)$ is a static dielectric function in random phase approximation and can be written as

$$
\kappa(q, 0)=1+\frac{q_{s}}{q} H(q)
$$

with $q_{s}=m^{*} e^{2} /\left(2 \pi \epsilon_{0} \kappa\right)$ and $H(q)$ given by ${ }^{35}$

$$
H(q)=3 \frac{1-\exp (-u)}{u^{2}+4 \pi^{2}}+\frac{u}{u^{2}+4 \pi^{2}}-\frac{1-\exp (-u)}{\left(u^{2}+4 \pi^{2}\right)^{2}}\left(u^{2}-4 \pi^{2}\right)+\frac{2}{u}\left[1-\frac{1-\exp (-u)}{u}\right] .
$$

Here, the effect of the Rashba SO coupling on the screening of $V_{\mathbf{q}}$ is ignored. Further, to determine the impurity density, we assume that for $M=0$ and $\alpha=0$ the electron-impurity scattering results in an electron mobility $\mu_{0}=5 \mathrm{~m}^{2} / \mathrm{Vs}$.

\section{A. Anomalous Hall effect in a InSb/AlInSb quantum well without Rashba SO coupling}

We first analyzed the anomalous Hall effect in the absence of Rashba SO interaction. In this case, the lowest order component of $J_{x}^{\mathrm{fd}}$ in $\lambda, J_{x}^{\mathrm{ss}-\mathrm{L}}$, vanishes, while the component $J_{x}^{\mathrm{ss}-\mathrm{F}}$ is nonvanishing and reduces to

$$
J_{x}^{\mathrm{ss}-\mathrm{F}}=e^{2} E \sum_{\mathbf{p}, \mu} \frac{p}{m^{*}} \cos ^{2} \phi_{\mathbf{p}} \Phi_{1 \mu}^{c}(p)
$$

with $\Phi_{1 \mu}^{c}(p)$ determined by

$$
\begin{aligned}
\Phi_{1 \mu}^{c}(p)= & (-1)^{\mu} 4 \pi^{2} n_{i} \tau_{1 \mu \mu} \lambda \Phi_{0 \mu}^{s}(p) \sum_{\mathbf{k}, \mathbf{q}} V_{\mathbf{p}-\mathbf{k}} V_{\mathbf{k}-\mathbf{q}} V_{\mathbf{q}-\mathbf{p}} p k \sin \left(\phi_{\mathbf{k}}-\phi_{\mathbf{p}}\right) \delta\left(\varepsilon_{\mu p}-\varepsilon_{\mu k}\right) \delta\left(\varepsilon_{\mu p}-\varepsilon_{\mu q}\right) \\
& \times\left[p k \sin \left(\phi_{\mathbf{k}}-\phi_{\mathbf{p}}\right)+q p \sin \left(\phi_{\mathbf{p}}-\phi_{\mathbf{q}}\right)-q k \sin \left(\phi_{\mathbf{k}}-\phi_{\mathbf{q}}\right)\right],
\end{aligned}
$$

and $\Phi_{0 \mu}^{s}(p)=-\tau_{1 \mu \mu} \frac{\partial n_{F}\left[\varepsilon_{\mu}(p)\right]}{\partial \varepsilon_{\mu}(p)} \frac{\partial \varepsilon_{\mu}(p)}{\partial p}$. Since the contributions to AHC from the off-diagonal elements of the distribution function, $J_{x}^{\text {fo }}=\left.J_{x}^{\text {fo }}\right|^{I}+\left.J_{x}^{\text {fo }}\right|^{I I}$, vanish, the side-jump AHC involves only the components $J_{x}^{\text {imp }}$ and $J_{x}^{E}: J_{x}^{\mathrm{sj}}=J_{x}^{\mathrm{imp}}+J_{x}^{E}$. Here, $J_{x}^{\text {imp }}$ and $J_{x}^{E}$ are equal to each other and take the form

$$
J_{x}^{\mathrm{imp}}=J_{x}^{E}=\lambda e^{2} E \sum_{\mathbf{p}}\left\{n_{F}\left[\varepsilon_{1}(p)\right]-n_{F}\left[\varepsilon_{2}(p)\right]\right\} .
$$

In Fig. 2, we plot the calculated total anomalous Hall conductivity $\sigma_{x y}=\sigma_{x y}^{\mathrm{ss}}+\sigma_{x y}^{\mathrm{sj}}$, and its component $\sigma_{x y}^{\mathrm{sj}}=$ $\sigma_{x y}^{\mathrm{imp}}+\sigma_{x y}^{E}$ as functions of magnetization, $M$. With increasing magnetization, $\sigma_{x y}$ and $\sigma_{x y}^{\mathrm{sj}}$ increase linearly. A 


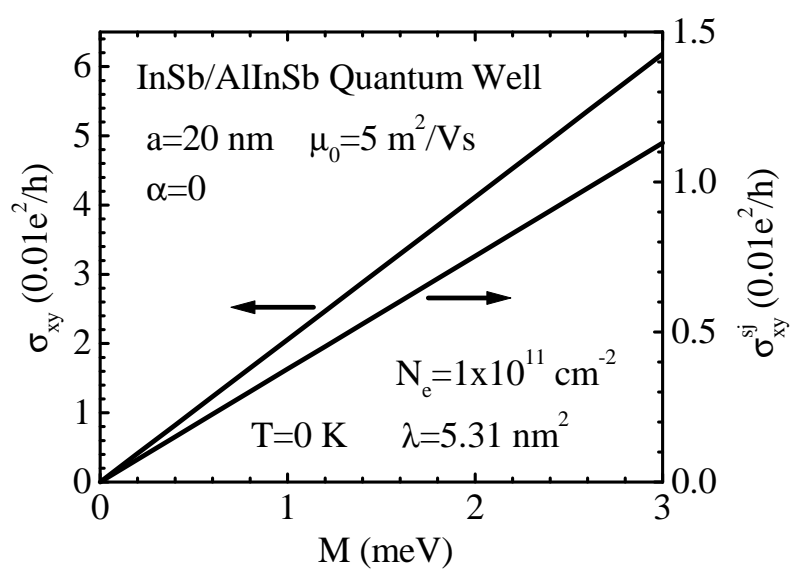

FIG. 2: Magnetization dependencies of $\sigma_{x y}$ and $\sigma_{x y}^{\mathrm{sj}}$ in a InSb/AlInSb quantum well without Rashba spin-orbit coupling. The width of the quantum well $a$ is $a=20 \mathrm{~nm}$. The electron density is $N_{e}=1 \times 10^{15} \mathrm{~m}^{-2}$. The lattice temperature is $T=0 \mathrm{~K}$ and the mobility in the absence of magnetization is $\mu_{0}=5 \mathrm{~m}^{2} / \mathrm{Vs}$.

comparison between $\sigma_{x y}$ and $\sigma_{x y}^{\mathrm{sj}}$ indicates that, for the given $\mu_{0}=5 \mathrm{~m}^{2} / \mathrm{Vs}$, both the contributions from side jump and skew scattering are of the same order of magnitude. Note that, notwithstanding the large spin-orbit coupling constant $\lambda$, the anomalous Hall conductivity is still much smaller than the ordinary one: the ordinary Hall conductivity is $34.6 e^{2} / h$ for a magnetic field $B=0.34 \mathrm{~T}$ (in the InSb/AlInSb quantum well with $g=-51.4$, this magnetic field corresponds to a magnetization $M=1 \mathrm{meV})$.

From Eqs. (65) and (66) we see that in the absence of both the Rashba SO coupling and magnetization, the contributions to anomalous Hall current from electrons with opposite spins (or helicities) have opposite signs. As a result, the total anomalous charge Hall current vanishes. However, there is a nonvanishing spin Hall current since electrons with opposite spins move toward opposite sides of the sample. We estimate the spin-Hall current in the studied InSb/AlInSb quantum well for $\alpha=0$ and $M=0$, finding that the spin-Hall mobility $\mu_{s H}$ defined in Ref. 36 is $\mu_{s H}=0.013 \mathrm{~m}^{2} / \mathrm{Vs}$. (In contrast to this, in a GaAs/AlGaAs quantum well with charge mobility $\mu_{0}=0.6 \mathrm{~m}^{2} / \mathrm{Vs}$, the total spin-Hall mobility is $\mu_{s H}=-2.0 \times 10^{-5} \mathrm{~m}^{2} / \mathrm{Vs}$, and the contributions from side-jump and skew scattering, respectively, are $\mu_{s H}^{s j}=-1.6 \times 10^{-4} \mathrm{~m}^{2} / \mathrm{Vs}$ and $\mu_{s H}^{s s}=1.4 \times 10^{-4} \mathrm{~m}^{2} / \mathrm{Vs}$. They are of the same order of magnitude as the spin-Hall mobilities in bulk $n$-doped GaAs: in a bulk GaAs with the same $\mu_{0}$, Engel et $a l^{25}$ found $\mu_{s H}^{s j}=-1.6 \times 10^{-4} \mathrm{~m}^{2} / \mathrm{Vs}$ and $\mu_{s H}^{s s}=3.5 \times 10^{-4} \mathrm{~m}^{2} / \mathrm{Vs}$.)

\section{B. Anomalous Hall effect in a Rashba InSb/AlInSb quantum well}

We have also calculated the anomalous Hall conductivity in a InSb/AlInSb quantum well with Rashba spin-orbit interaction. In the case of nonvanishing $\alpha$, one has to consider not only $\sigma_{x y}^{\mathrm{ss}-\mathrm{F}}$ and $\sigma_{x y}^{\mathrm{imp}, E}$, but also the anomalous Hall conductivities, $\sigma_{x y}^{\mathrm{fo}}=\left.\sigma_{x y}^{\mathrm{fo}}\right|^{I}+\left.\sigma_{x y}^{\mathrm{fo}}\right|^{I I}$, and $\sigma_{x y}^{\text {ss }-\mathrm{L}}$. The results are plotted in Figs. 3 and 4.

In Fig. 3, we plot the total anomalous Hall conductivity, $\sigma_{x y}$, and the skew scattering and side-jump Hall conductivities, $\sigma_{x y}^{\text {ss }}$ and $\sigma_{x y}^{\text {sj }}$, as well as their components, $\sigma_{x y}^{\text {ss-L }}, \sigma_{x y}^{\text {fo }}, \sigma_{x y}^{E}$, and $\sigma_{x y}^{\text {imp }}$, as functions of magnetization $M$ for various Rashba spin-orbit coupling constants. We find that, as magnetization increases, the total anomalous Hall conductivity $\sigma_{x y}$ increases for $\alpha=5,10$, and $20 \mathrm{meV} \cdot \mathrm{nm}$, but it decreases for $\alpha=50 \mathrm{meV} \cdot \mathrm{nm}$.

Such complicated behavior of the magnetization dependence of $\sigma_{x y}$ arises from competition of the side-jump and skew-scattering contributions to anomalous Hall conductivity. From Figs. 3(b) and 3(c) it is obvious that $\sigma_{x y}^{\text {ss }}$ varies monotonically with the magnetization, but in the magnetization dependence of $\sigma_{x y}^{\mathrm{sj}}$ there is always a small dip (when $\alpha=50 \mathrm{meV} \cdot \mathrm{nm}$, this dip is shifted out of the studied magnetization range). In $\sigma_{x y}^{\text {ss }}, \sigma_{x y}^{\text {ss }-\mathrm{F}}$ is dominant for small $\alpha$, leading to an increase of $\sigma_{x y}^{\mathrm{ss}}$ with increasing $M$. However, when $\alpha>30 \mathrm{meV} \cdot \mathrm{nm}, \sigma_{x y}^{\mathrm{ss}-\mathrm{L}}$ is important: $\sigma_{x y}^{\mathrm{ss}}$ becomes negative and its magnetization dependence exhibits a decrease with increasing magnetization. Among the contributions to $\sigma_{x y}^{\mathrm{sj}}, \sigma_{x y}^{\mathrm{fo}}$ is dominant for large magnetization, while $\sigma_{x y}^{E}$ is important for small $M$ and results in a nonvanishing $\sigma_{x y}^{\text {sj }}$ when $\alpha \neq 0$ but $M=0$.

In Fig. 4, we plot $\sigma_{x y}, \sigma_{x y}^{\mathrm{ss}}$, and $\sigma_{x y}^{\mathrm{sj}}$ as functions of the spin-orbit coupling constant. We find that, as $\alpha$ increases, $\sigma_{x y}$ increases monotonically for $M=0 \mathrm{meV}$, while for $M=0.5-2 \mathrm{meV}, \sigma_{x y}$ first decreases and then increases. It is also evident from Figs. 4(b) and 4(c) that, with increasing Rashba spin-orbit coupling constant $\alpha, \sigma_{x y}^{\text {sj }}$ increases while 

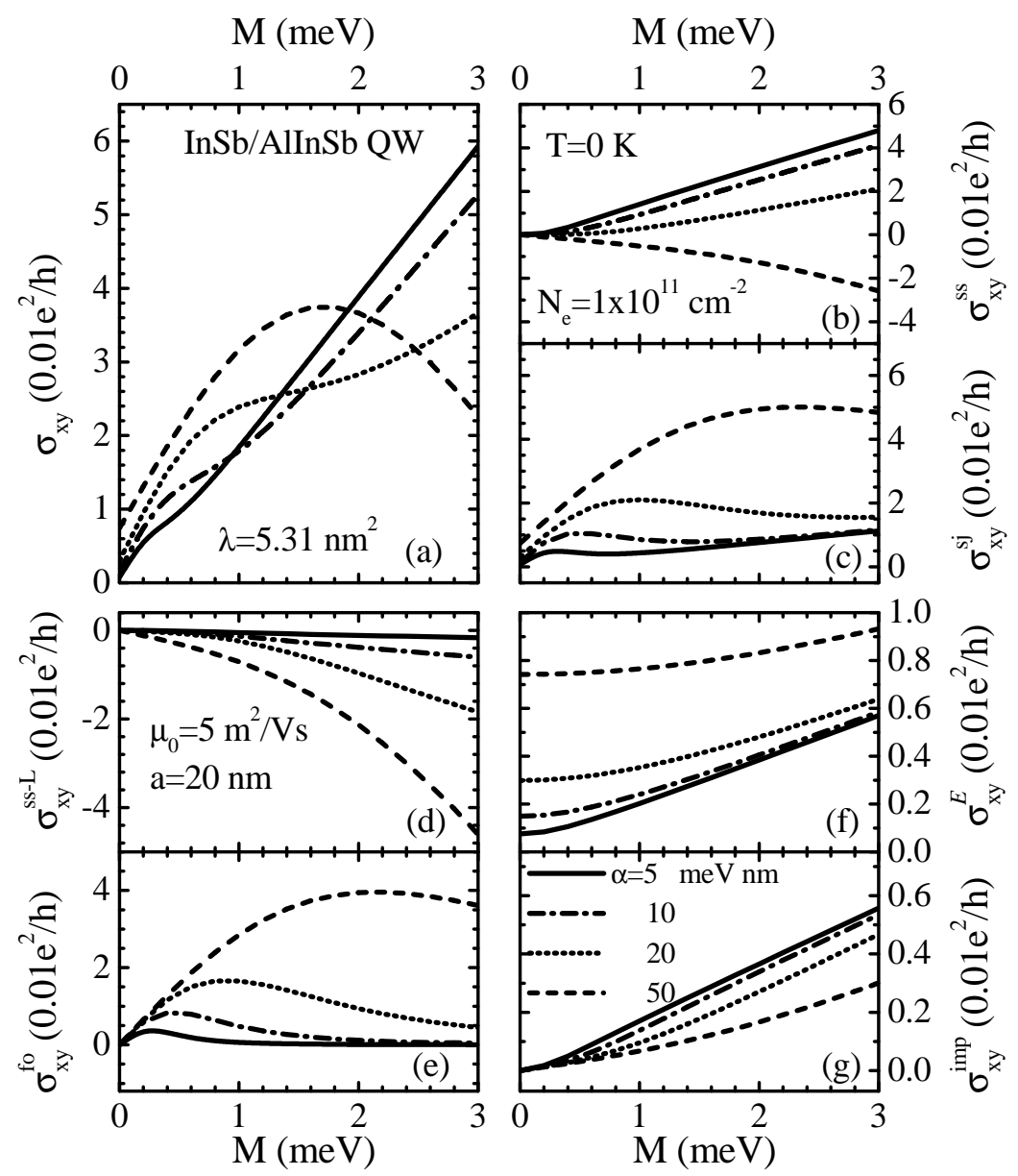

FIG. 3: Magnetization dependencies of (a) the total anomalous Hall conductivity, $\sigma_{x y}$, (b) the skew-scattering and (c) side-jump Hall conductivities, as well as their components, (d) $\sigma_{x y}^{\text {ss }-\mathrm{L}}$, (e) $\sigma_{x y}^{\text {fo }}$, (f) $\sigma_{x y}^{E}$, and (g) $\sigma_{x y}^{\text {imp }}$ in a InSb/AlInSb quantum well with different Rashba SO couplings: $\alpha=5,10,20$, and $50 \mathrm{meV} \cdot \mathrm{nm}$. The other parameters are the same as in Fig. 2.

$\sigma_{x y}^{\text {ss }}$ decreases. Since the rate of increase or decrease depends on $M$, the $\alpha$ dependence of the total anomalous Hall conductivity behaves differently for different magnetizations.

\section{CONCLUSIONS}

We have employed a kinetic equation approach to investigate the anomalous Hall effect in Rashba 2D electron systems based on narrow band semiconductors. The Rashba SO coupling was considered nonperturbatively, while the extrinsic spin-orbit interaction and the SO coupling directly induced by an external driving electric field were taken into account in the first order of the coupling constant. Considering electron-impurity scattering up to the secondBorn approximation, we found that the various components of the anomalous Hall current can fit into two classes: the side-jump and skew scattering anomalous Hall currents. The side-jump anomalous Hall current involves contributions not only from the extrinsic SO coupling, but also from SO coupling directly induced by the driving electric field. It also contains a component which arises from Rashba SO coupling and relates to the off-diagonal elements of the helicity-basis distribution function. The skew-scattering AHE arises from the anisotropy of the diagonal elements of the distribution function, and it is a result of the Rashba and extrinsic SO interactions. We also performed a numerical calculation to investigate the anomalous Hall effect in a InSb/AlInSb quantum well. We found that the contributions to anomalous Hall conductivity from both the side-jump and skew scattering terms are of the same order of magnitude, leading to complicated dependencies of the total anomalous Hall conductivity on magnetization and on the Rashba spin-orbit coupling constant. It is also clear that the component arising from the SO coupling due to the driving electric field is dominant for small magnetization. 


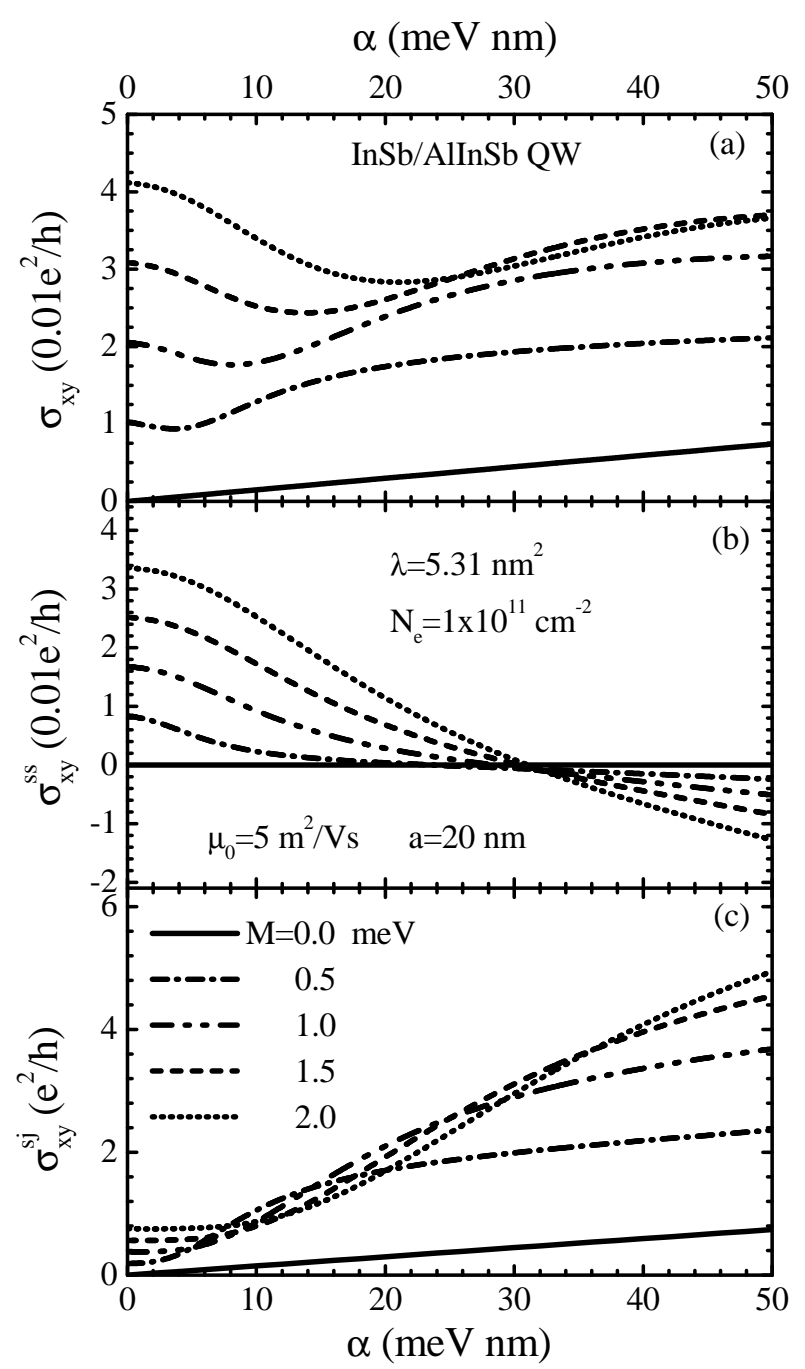

FIG. 4: The dependencies of (a) the total anomalous Hall conductivity $\sigma_{x y}$, and (b) the skew-scattering and (c) the side-jump anomalous Hall conductivities, $\sigma_{x y}^{\mathrm{ss}}$ and $\sigma_{x y}^{\mathrm{sj}}$, on the Rashba spin-orbit coupling constant for different magnetizations: $M=0$, 0.5, 1.0, 1.5, and $2.0 \mathrm{meV}$. The other parameters are the same as in Fig. 2.

\section{Acknowledgments}

This work was supported by the Department of Defense through the DURINT program administered by the US Army Research Office, DAAD Grant No. 19-01-1-0592, and by projects of the National Science Foundation of China and the Shanghai Municipal Commission of Science and Technology.

\section{APPENDIX: EXPLICIT FORM OF $\hat{I}^{(1)}$}

The diagonal elements of $\hat{I}^{(1)},\left(\hat{I}^{(1)}\right)_{\mu \mu}$, can be written as sums of four terms: $\left(\hat{I}^{(1)}\right)_{\mu \mu}=\mathcal{I}_{\mu}^{a}\left[\hat{\rho}_{1}^{I}\right]+\mathcal{I}_{\mu}^{b}\left[\hat{\rho}_{1}^{I}\right]+\mathcal{I}_{\mu}^{c}\left[\hat{\rho}_{1}^{I}\right]+$ $\mathcal{I}_{\mu}^{d}\left[\hat{\rho}_{1}^{I}\right]\left(\left[\hat{\rho}_{1}^{I}\right]\right.$ denotes that $\mathcal{I}_{\mu}^{a, b, c, d}$ depend on the specific form of the distribution function $\left.\hat{\rho}_{1}^{I}\right)$. $\mathcal{I}_{\mu}^{a}\left[\hat{\rho}_{1}^{I}\right]$ and $\mathcal{I}_{\mu}^{b}\left[\hat{\rho}_{1}^{I}\right]$ are the terms of the first Born approximation and can be written as

$$
\begin{aligned}
\mathcal{I}_{\mu}^{a}\left[\hat{\rho}_{1}^{I}\right]= & \pi n_{i} \sum_{\mathbf{k}}|V(\mathbf{p}-\mathbf{k})|^{2}\left\{a_{+}(\mathbf{p}, \mathbf{k})\left[\left(\rho_{1}^{I}\right)_{\mu \mu}(\mathbf{p})-\left(\rho_{1}^{I}\right)_{\mu \mu}(\mathbf{k})\right] \Delta_{\mu \mu}\right. \\
& \left.+a_{-}(\mathbf{p}, \mathbf{k})\left[\left(\rho_{1}^{I}\right)_{\mu \mu}(\mathbf{p})-\left(\rho_{1}^{I}\right)_{\bar{\mu} \bar{\mu}}(\mathbf{k})\right] \Delta_{\mu \bar{\mu}}\right\}
\end{aligned}
$$


and

$$
\begin{aligned}
\mathcal{I}_{\mu}^{b}\left[\hat{\rho}_{1}^{I}\right]= & 2 \pi n_{i} \lambda \alpha^{2} \sum_{\mathbf{k}}|V(\mathbf{p}-\mathbf{k})|^{2} \frac{k^{2} p^{2}}{\lambda_{p} \lambda_{k}} \sin ^{2}\left(\phi_{\mathbf{k}}-\phi_{\mathbf{p}}\right) \\
& \times\left\{\left[\left(\rho_{1}^{I}\right)_{\mu \mu}(\mathbf{p})-\left(\rho_{1}^{I}\right)_{\mu \mu}(\mathbf{k})\right] \Delta_{\mu \mu}-\left[\left(\rho_{1}^{I}\right)_{\mu \mu}(\mathbf{p})-\left(\rho_{1}^{I}\right)_{\bar{\mu} \bar{\mu}}(\mathbf{k})\right] \Delta_{\mu \bar{\mu}}\right\},
\end{aligned}
$$

with $\Delta_{\mu \nu} \equiv \delta\left[\varepsilon_{\mu}(\mathbf{p})-\varepsilon_{\nu}(\mathbf{k})\right]$ and $a_{ \pm}(\mathbf{p}, \mathbf{k}) \equiv\left(\lambda_{p} \lambda_{k} \pm M^{2} \pm \alpha^{2} k p \cos \left(\phi_{\mathbf{p}}-\phi_{\mathbf{k}}\right)\right) / \lambda_{p} \lambda_{k} . \mathcal{I}_{\mu}^{c}\left[\hat{\rho}_{1}^{I}\right]$ and $\mathcal{I}_{\mu}^{d}\left[\hat{\rho}_{1}^{I}\right]$ are the terms of the second Born approximation. $\mathcal{I}_{\mu}^{c}\left[\hat{\rho}_{1}^{I}\right]$ is explicitly independent of the spin-orbit coupling constant $\lambda$ and takes the form

$$
\begin{aligned}
\mathcal{I}_{\mu}^{c}\left[\hat{\rho}_{1}^{I}\right]= & \pi^{2} M n_{i} \alpha^{2} \sum_{\mathbf{k}, \mathbf{q}} V_{\mathbf{p}-\mathbf{k}} V_{\mathbf{k}-\mathbf{q}} V_{\mathbf{q}-\mathbf{p}} \frac{1}{\lambda_{p} \lambda_{k}} \\
& \times\left\{c_{\mu+}(\mathbf{p}, \mathbf{q}, \mathbf{k}) \Delta_{\mu \mu}\left(\rho_{1}^{I}\right)_{\mu \mu}(\mathbf{k})+c_{\mu-}(\mathbf{p}, \mathbf{q}, \mathbf{k}) \Delta_{\mu \bar{\mu}}\left(\rho_{1}^{I}\right)_{\bar{\mu} \bar{\mu}}(\mathbf{k})\right\}
\end{aligned}
$$

while $\mathcal{I}_{\mu}^{d}\left[\hat{\rho}_{1}^{I}\right]$ is linear in $\lambda$ and can be written as

$$
\begin{aligned}
\mathcal{I}_{\mu}^{d}\left[\hat{\rho}_{1}^{I}\right]= & \pi^{2} \lambda M n_{i} \sum_{\mathbf{k}, \mathbf{q}} V_{\mathbf{p}-\mathbf{k}} V_{\mathbf{k}-\mathbf{q}} V_{\mathbf{q}-\mathbf{p}} \frac{1}{\lambda_{p} \lambda_{k}} \\
& \times\left\{d_{\mu+}(\mathbf{p}, \mathbf{q}, \mathbf{k}) \Delta_{\mu \mu}\left(\rho_{1}^{I}\right)_{\mu \mu}(\mathbf{k})+d_{\mu-}(\mathbf{p}, \mathbf{q}, \mathbf{k}) \Delta_{\mu \bar{\mu}}\left(\rho_{1}^{I}\right)_{\bar{\mu} \bar{\mu}}(\mathbf{k})\right\}
\end{aligned}
$$

The parameters $c_{\mu \pm}(\mathbf{p}, \mathbf{k}, \mathbf{q})$ and $d_{\mu \pm}(\mathbf{p}, \mathbf{k}, \mathbf{q})$ are defined as

$$
\begin{aligned}
c_{\mu \pm}(\mathbf{p}, \mathbf{q}, \mathbf{k})= & \mp \frac{(-1)^{\mu}}{\lambda_{q}} \mathcal{C}(\mathbf{p}, \mathbf{q}, \mathbf{k})\left[\delta\left(\varepsilon_{\mu}(\mathbf{p})-\varepsilon_{\mu}(\mathbf{q})\right)-\delta\left(\varepsilon_{\mu}(\mathbf{p})-\varepsilon_{\bar{\mu}}(\mathbf{q})\right)\right] \\
d_{\mu \pm}(\mathbf{p}, \mathbf{k}, \mathbf{q})= & \frac{1}{\lambda_{q}}\left\{\mathcal{D}_{\mu \pm}^{m}(\mathbf{p}, \mathbf{q}, \mathbf{k})\left[\delta\left(\varepsilon_{\mu}(\mathbf{p})-\varepsilon_{\mu}(\mathbf{q})\right)-\delta\left(\varepsilon_{\mu}(\mathbf{p})-\varepsilon_{\bar{\mu}}(\mathbf{q})\right)\right]\right. \\
& \left.+\mathcal{D}_{\mu \pm}^{p}(\mathbf{p}, \mathbf{q}, \mathbf{k})\left[\delta\left(\varepsilon_{\mu}(\mathbf{p})-\varepsilon_{\mu}(\mathbf{q})\right)+\delta\left(\varepsilon_{\mu}(\mathbf{p})-\varepsilon_{\bar{\mu}}(\mathbf{q})\right)\right]\right\}
\end{aligned}
$$

with

$$
\begin{aligned}
& \mathcal{C}(\mathbf{p}, \mathbf{q}, \mathbf{k})=p q \sin \left(\phi_{\mathbf{p}}-\phi_{\mathbf{q}}\right)-q k \sin \left(\phi_{\mathbf{k}}-\phi_{\mathbf{q}}\right)+p k \sin \left(\phi_{\mathbf{k}}-\phi_{\mathbf{p}}\right) \\
\mathcal{D}_{\mu \pm}^{m}(\mathbf{p}, \mathbf{q}, \mathbf{k})= & (-1)^{\mu+1}\left( \pm M^{2}+\lambda_{k} \lambda_{p}\right)\left[p k \sin \left(\phi_{\mathbf{k}}-\phi_{\mathbf{p}}\right)+q p \sin \left(\phi_{\mathbf{p}}-\phi_{\mathbf{q}}\right)-q k \sin \left(\phi_{\mathbf{k}}-\phi_{\mathbf{q}}\right)\right] \\
& \pm(-1)^{\mu+1} \alpha^{2}\left\{p k \sin \left(\phi_{\mathbf{k}}-\phi_{\mathbf{p}}\right)\left[p q \cos \left(\phi_{\mathbf{p}}-\phi_{\mathbf{q}}\right)+q k \cos \left(\phi_{\mathbf{q}}-\phi_{\mathbf{k}}\right)-k p \cos \left(\phi_{\mathbf{k}}-\phi_{\mathbf{p}}\right)\right]\right. \\
& +q p \sin \left(\phi_{\mathbf{p}}-\phi_{\mathbf{q}}\right)\left[-p q \cos \left(\phi_{\mathbf{p}}-\phi_{\mathbf{q}}\right)+q k \cos \left(\phi_{\mathbf{q}}-\phi_{\mathbf{k}}\right)+k p \cos \left(\phi_{\mathbf{k}}-\phi_{\mathbf{p}}\right)\right] \\
& \left.+q k \sin \left(\phi_{\mathbf{k}}-\phi_{\mathbf{q}}\right)\left[-p q \cos \left(\phi_{\mathbf{p}}-\phi_{\mathbf{q}}\right)+q k \cos \left(\phi_{\mathbf{q}}-\phi_{\mathbf{k}}\right)-k p \cos \left(\phi_{\mathbf{k}}-\phi_{\mathbf{p}}\right)\right]\right\}
\end{aligned}
$$

and

$$
\mathcal{D}_{\mu \pm}^{p}(\mathbf{p}, \mathbf{q}, \mathbf{k})=(-1)^{\mu+1} \lambda_{q}\left(\lambda_{k} \pm \lambda_{p}\right)\left[p k \sin \left(\phi_{\mathbf{k}}-\phi_{\mathbf{p}}\right)+q p \sin \left(\phi_{\mathbf{p}}-\phi_{\mathbf{q}}\right)-q k \sin \left(\phi_{\mathbf{k}}-\phi_{\mathbf{q}}\right)\right] .
$$

Since the off-diagonal elements of the collision term $\hat{I}^{(1)}$ are simply related by $\hat{I}_{12}^{(1)}=-\left[\hat{I}_{12}^{(1)}\right]^{*}$, it suffices to consider the element $\hat{I}_{12}^{(1)}$. In the first-Born approximation, $\hat{I}_{12}^{(1)}$ can be expressed as a sum of two terms: $\hat{I}_{12}^{(1)}=\mathcal{S}^{a}\left[\hat{\rho}_{1}^{I}\right]+\mathcal{S}^{b}\left[\hat{\rho}_{1}^{I}\right]$ with $\mathcal{S}^{a}\left[\hat{\rho}_{1}^{I}\right]$ and $\mathcal{S}^{b}\left[\hat{\rho}_{1}^{I}\right]$ as the terms in the lowest- and first-order of $\lambda$, respectively, and determined by

$$
\begin{aligned}
\mathcal{S}^{a, b}\left[\hat{\rho}_{1}^{I}\right]= & \frac{n_{i}}{2} \sum_{\mathbf{k}}|V(\mathbf{p}-\mathbf{k})|^{2} g_{a, b}(\mathbf{p}, \mathbf{k})\left\{\left[\left(\hat{\mathrm{G}}_{0 k}^{r}\right)_{11}\left(\varepsilon_{2 p}\right)-\left(\hat{\mathrm{G}}_{0 k}^{r}\right)_{22}\left(\varepsilon_{2 p}\right)\right]\left(\hat{\rho}_{1}^{I}\right)_{22}(\mathbf{p})\right. \\
& -\left[\left(\hat{\mathrm{G}}_{0 p}^{a}\right)_{22}\left(\varepsilon_{2 k}\right)-\left(\hat{\mathrm{G}}_{0 p}^{r}\right)_{11}\left(\varepsilon_{2 k}\right)\right]\left(\hat{\rho}_{1}^{I}\right)_{22}(\mathbf{k})-\left[\left(\hat{\mathrm{G}}_{0 k}^{a}\right)_{11}\left(\varepsilon_{1 p}\right)-\left(\hat{\mathrm{G}}_{0 k}^{a}\right)_{22}\left(\varepsilon_{1 p}\right)\right]\left(\hat{\rho}_{1}^{I}\right)_{11}(\mathbf{p}) \\
& \left.+\left[\left(\hat{\mathrm{G}}_{0 p}^{a}\right)_{22}\left(\varepsilon_{1 k}\right)-\left(\hat{\mathrm{G}}_{0 p}^{r}\right)_{11}\left(\varepsilon_{1 k}\right)\right]\left(\hat{\rho}_{1}^{I}\right)_{11}(\mathbf{k})\right\} .
\end{aligned}
$$


Here, $g_{a}(\mathbf{p}, \mathbf{k}) \equiv\left[\alpha k \lambda_{p} \sin \left(\phi_{\mathbf{p}}-\phi_{\mathbf{k}}\right)+i \alpha M\left(p-k \cos \left(\phi_{\mathbf{p}}-\phi_{\mathbf{k}}\right)\right)\right] /\left(\lambda_{k} \lambda_{p}\right)$ and $g_{b}(\mathbf{p}, \mathbf{k}) \equiv-2 \sin \left(\phi_{\mathbf{p}}-\phi_{\mathbf{k}}\right)\left[i M \sin \left(\phi_{\mathbf{p}}-\right.\right.$ $\left.\left.\left.\phi_{\mathbf{k}}\right)+\lambda_{p} \cos \left(\phi_{\mathbf{p}}-\phi_{\mathbf{k}}\right)\right)\right] \lambda \alpha k^{2} p /\left(\lambda_{k} \lambda_{p}\right)$.

* Electronic address: liusy@mail.sjtu.edu.cn

1 C. M. Hurd, The Hall Effect in Metals and Alloys (Plenum Press, New York, 1972); L. Berger and G. Bergmann, in The Hall Effect and Its Applications edited by C. L. Chien and C. R. Westgate (Plenum, New York, 1979).

2 E. H. Hall, Phil. Mag. 12, 157 (1881).

${ }^{3}$ For a recent review, see J. Sinova, T. Jungwirth, and J. Černe, Int. J. Mod. Phys. B 18, 1083 (2004).

${ }^{4}$ For a review, see R. H. Silsbee, J. Phys.: Condens. Matter 16, R179(2004).

5 R. Karplus and J. M. Luttinger, Phys. Rev. 95, 1154 (1954); J. M. Luttinger, Phys. Rev. 112, 739 (1958).

6 T. Jungwirth, Q. Niu, and A. H. MacDonald, Phys. Rev. Lett. 88, 207208 (2002).

7 T. Jungwirth, J. Sinova, K. Wang, K. W. Edmonds, R. Campion, B. Gallagher, C. Foxon, Q. Niu, and A. H. MacDonald, Appl. Phys. Lett. 83, 320 (2003).

8 Y. Yao, L. Kleinman, A. H. MacDonald, J. Sinova, T. Jungwirth, D. S. Wang, E. Wang, and Qian Niu, Phys. Rev. Lett. 92, 037204 (2004).

9 Z. Fang, N. Nagaosa, K. S. Takahashi, A. Asamitsu, R. Mathieu, T. Ogasawara, H. Yamada, M. Kawasaki, Y. Tokura, and K. Terakura, Science 302,92 (2003).

10 R. Mathieu, A. Asamitsu, H. Yamada, K. S. Takahashi, M. Kawasaki, Z. Fang, N. Nagaosa, and Y. Tokura, Phys. Rev. Lett. 93, 016602 (2004).

11 R. Mathieu, C. U. Jung, H. Yamada, A. Asamitsu, M. Kawasaki, and Y. Tokura, Phys. Rev. B 72, 064436 (2005).

12 W.-L. Lee, S. Watauchi, V. L. Miller, R. J. Cava, and N. P. Ong, Science 303, 1647 (2004).

13 L. Berger, Phys. Rev. B 2, 4559 (1970); 5, 1862 (1972).

14 J. Smit, Physica 21, 877 (1955); ibid. 24, 39 (1958).

15 N. A. Sinitsyn, Q. Niu, and A. H. MacDonald, Phys. Rev. B 73, 075318 (2006).

16 P. Leroux-Hugon and A. Ghazali, J. Phys. C: Solid State Phys. 5, 1072(1972).

17 A. Crépieux and P. Bruno, Phys. Rev. B 64, 014416 (2001).

18 V. K. Dugaev, A. Crépieux, and P. Bruno, Phys. Rev. B 64, 104411(2001).

19 A. Crépieux, J. Wunderlich, V. K. Dugaev, and P. Bruno, J. Magn. Magn. Mater. 242-245, 464 (2002).

20 A. Langenfeld and P. Wölfle, Phys. Rev. Lett. 67, 739 (1991).

21 P. Wölfle and K. A. Muttalib, cond-mat/0510481 (unpublished).

22 N.A. Sinitsyn, Qian Niu, J. Sinova, and K. Nomura, Phys. Rev. B 72, 045346 (2005).

${ }^{23}$ V. K. Dugaev, P. Bruno, M. Taillefumier, B. Canals, and C. Lacroix, Phys. Rev. B 71, 224423 (2005).

24 S. Y. Liu and X. L. Lei, Phys. Rev. B 72, 195329(2005).

25 H.-A. Engel, B. I. Halperin, and E. I. Rashba, Phys. Rev. Lett. 95, 166605 (2005).

26 P. Nozières and C. Lewiner, J. Phys. (Paris) 34, 901 (1973).

27 R. Winkler, Spin-Orbit Coupling Effects in Two-Dimensional Electron and Hole System (Springer, New York, 2003).

28 G. D. Mahan, Many-Particle Physics (Plenum, NY, 1990).

29 P. Lipavský, V. Špička, and B. Velicky, Phys. Rev. B 34, 6933 (1986).

30 H. Haug, Phys. Status Solidi (b) 173, 139 (1992).

31 H. Haug and A.-P. Jauho, Quantum Kinetics in Transport and Optics of Semiconductors (Springer, 1996).

32 G. A. Khodaparast, R. E. Doezema, S. J. Chung, K. J. Goldammer, and M. B. Santos, Phys. Rev. B 70, 155322 (2004).

33 J.-M. Jancu, R. Scholz, E. A. de Andrada e Silva, and G. C. La Rocca, Phys. Rev. B 72, 193201 (2005).

34 X. L. Lei, N. J. M. Horing, and J. Q. Zhang, Phys. Rev. B 34, 1139 (1986).

35 P. J. Price, Phys. Rev. B 30, 2234 (1984).

36 S. Y. Liu, Norman J. M. Horing, and X. L. Lei, Phys. Rev. B 73, 205207 (2006). 